\title{
Sample Preparation in the Analysis of Pesticides Residue in Food by Chromatographic Techniques
}

\author{
Guan Huat Tan ${ }^{1}$ and Mee-Kin Chai ${ }^{2}$ \\ ${ }^{1}$ Department of Chemistry, University of Malaya, 50603 Kuala Lumpur \\ ${ }^{2}$ Department of Engineering Science and Mathematics, College of Engineering, \\ Universiti Tenaga Nasional, Km 7 Jalan Kajang-Puchong, 43009 Kajang, Selangor \\ Malaysia
}

\section{Introduction}

Food samples present an enormous challenge to analytical chemists in their efforts to determine residues of pesticides at trace levels to satisfy food safety regulations in EU, USA and Japan. The wide array of food matrices from liquids to solids require different sample preparation techniques for accurate and reproducible results with chromatographic techniques such as Gas chromatography(GC) and High Performance Liquid Chromatography (HPLC). In addition, there exists a wide range of pesticides which are used legally for crop protection and their residue content in food must be accurately monitored for safe consumption. The GC and HPLC techniques with different types of detector systems can provide such analysis at trace levels to fulfill the maximum residue levels(MRL) as per the food safety regulations in these countries. However, the accurate and reproducible results often depend upon the sample preparation techniques associated with the different food matrices.

Sample preparation has always been regarded as the bottleneck in the analytical laboratory performing numerous analyses, but it is the key to accurate analysis. In this regard, as per pesticide residue analysis, where not only the physical volume of the analyses can be enormous but also the number of pesticides involved can range from a selected few to a broad spectrum depending on the food source. This will usually necessitate the employment of different sample preparation methods for different targeted pesticides as well as the multitude of food matrices.

It has been estimated that the sample preparation step in most determinations consume approximately $60-70 \%$ of the total time required for the analysis. It must be able to produce analytically accurate results and be economically efficient for routine analysis. In addition, it must be safe and easy to perform.

Most sample preparation procedures for GC and HPLC determination follow the basic steps as outlined below:

1. The food sample is homogenized or blended to obtain a uniform matrix.

2. This will be followed by extraction of the pesticide residue with solvents.

3. A cleanup step is employed to remove interfering matrix components from the GC or HPLC chromatograms. 
4. The elution and/or fractionation of the extracted analytes.

5. Concentrate the eluent and re-constitute in a solvent which is compatible with the GC or HPLC conditions.

6. Finally, the solution containing the pesticide can be introduced into the GC or HPLC.

The different types of sample preparation for such analyses will be presented. These are liquid-liquid extraction, solid phase extraction, solid phase microextraction, single drop microextraction, liquid-solid extraction, microwave assisted solvent extraction, supercritical fluid extraction, dispersive liquid-liquid microextraction and accelerated solvent extraction.

\section{Liquid-liquid Extraction (LLE)}

Analytes in solutions or liquid samples can be extracted by direct partitioning with an immiscible solvent. Liquid-liquid extraction (LLE) is based on the relative solubility of an analyte in two immiscible phases and is governed by the equilibrium distribution/partition coefficient. Extraction of an analyte is achieved by the differences in the solubilising power (polarity) of the two immiscible liquid phases.

LLE is traditionally one of the most common methods of extraction, particularly for organic compounds from aqueous matrices. Typically a separating funnel is used and the two immiscible phases are mixed by shaking and then allowed to separate. To avoid emulsions, in some cases, a salt may be added and centrifugation can be used if necessary. Alternatively a matrix solid-phase dispersion(MSPD) approach can be used to avoid emulsions. Both layers can be collected for further analysis. To ensure the complete extraction of an analyte into the required phase, multiple extractions may be necessary. Due to the limited selectivity, particularly for trace level analysis, there is a need for cleanup or analyte enrichment and concentration steps prior to instrumental analysis.

In the case of multiresidue methods, the extracting solvent has to be suitable for the extraction of compounds within a wide polarity range from a variety of matrices containing different amounts of water, fats, sugars and other substances. The usual way for extracting pesticide residues from the sample is by thorough disintegration of the matrix in a high speed homogenizer in the presence of the solvent or solvent mixture. In this way, even the AOAC method, which is one of the most commonly instituted methods, has been modified. The original methods which were extraction with acetonitrile, followed by liquid-liquid partitioning with petroleum ether/dichloromethane and a laborious florisil column cleanup, was modified in 1985 to include acetone instead of acetonitrile (Torres et al., 1996).

Acetone extraction is usually preferred since it is suitable for both non-polar and polar pesticides, as has been demonstrated in many comparative studies performed by GC and HPLC. In addition, acetone has low toxicity, is easy to purify, evaporate and filter and is inexpensive. Fruit and vegetable extracts in acetone are usually cleaner than those obtained with other solvents of similar polarity (Torres et al., 1996).

A rapid and efficient multiresidue extraction procedure using ethyl acetate and sodium sulphate, followed by GPC on an SX-3 column, was first reported by Roos et al. (1987). Recoveries better than $90 \%$ were obtained for organochlorine(OC) and organophophorous (OP) pesticides, fungicides and chlorobiphenyls. The ethyl acetate and sodium sulphate extraction without further cleanup was applied as a screening method for the analysis of eight OP pesticides with varying polarities in different types of vegetables using gas chromatography coupled to the flame photometric and nitrogen-phosphorous(GC-FPD, GCNPD) detectors. With the use of specific detectors, interfering chromatographic peaks were 
reduced and the analysis time and solvent usage were also minimized, resulting in lowercost analyses (Cai et al., 1995).

In another study, Castro et al. (2002) developed a rapid LLE method for the determination of endosulfan isomers and endosulfan-sulfate in plant samples. Tomato leaf samples were homogenized with ethyl acetate and extracts cleaned-up on an aluminium oxide column. The pesticides were eluted with a hexane-ethyl acetate (80:20, v/v) mixture. Recoveries obtained from plant samples were higher than $78 \%$ with an RSD lower than $14 \%$ and detection limits were $0.02 \mu \mathrm{g} / \mathrm{g}$ for each pesticide. Barriada-Pereira et al. (2004) compared the use of cartridges filled with four different sorbents: florisil, a tandem of florisil and alumina, silica, and carbon black to clean up plant leaf extracts prior to OC pesticides determination. Carbon black was shown to be the preferred sorbent, providing colorless eluates, cleaner chromatograms and fewer interferences. Jansson et al. (2004) used the National Food Administration (NFA) ethyl acetate extraction to determine 57 different pesticides and metabolites in a wide variety of fruits and vegetables by LC-MS/MS. The recoveries obtained were in the range $70-100 \%$. The proposed method is quick and straightforward and no additional clean-up steps are needed.

\section{Solid-phase Extraction (SPE)}

Solid phase extraction (SPE) was developed in the mid-1970 as an alternative approach to LLE for separation, purification, pre-concentration and solvent exchange of solutes for solution (Thurman and Mills, 1998). SPE can be used directly as an extraction technique for liquid matrices, or as a cleanup method for solvent extracts.

An SPE method always consists of three to four successive steps, as illustrated in Figure 1. First, the solid sorbent should be conditioned using an appropriate solvent. This step is crucial, as it enables the wetting of the packing material and the solvation of the functional groups. In addition, it removes possible impurities initially contained in the sorbent or the packaging. Also, this step removes the air present in the column and fills the void volume with solvent. The nature of the conditioning solvent depends on the type of the solid sorbent. Typically, for reversed phase sorbent, methanol is frequently used, followed by water or an aqueous buffer whose $\mathrm{pH}$ and ionic strength are similar to that of the sample. Precautionary steps are taken to prevent the solid sorbent from drying in between the conditioning and the sample treatment steps, otherwise the analytes will not be efficiently retained giving rise to poor recoveries. If the sorbent is dry for more than several minutes, it must be reconditioned.

The second step is the percolation of the sample through the solid sorbent. Depending on the system used, the volumes used can range from $1 \mathrm{~mL}$ to $1 \mathrm{~L}$. The sample may be applied to the column by gravity, pumping, aspirated by vacuum or by an automated system. The sample flow rate through the sorbent should be low enough to enable efficient retention of the analytes, and high enough to avoid excessive retention. During this step, the analytes are concentrated on the sorbent. Even though the matrix components may also be retained by the solid sorbent, some of them could pass through, thus enabling some purification (matrix separation) of the sample.

The third step (which is optional) may be the washing of the solid sorbent with an appropriate solvent, having low elution strength, to eliminate matrix components which have been retained by the solid sorbent, without displacing the analytes. A drying step may also be advisable, especially for aqueous matrices, to remove traces of water from the solid 
sorbent. This will eliminate the presence of water in the final extract, which, in some cases, may hinder the subsequent concentration of the extract and the analysis.

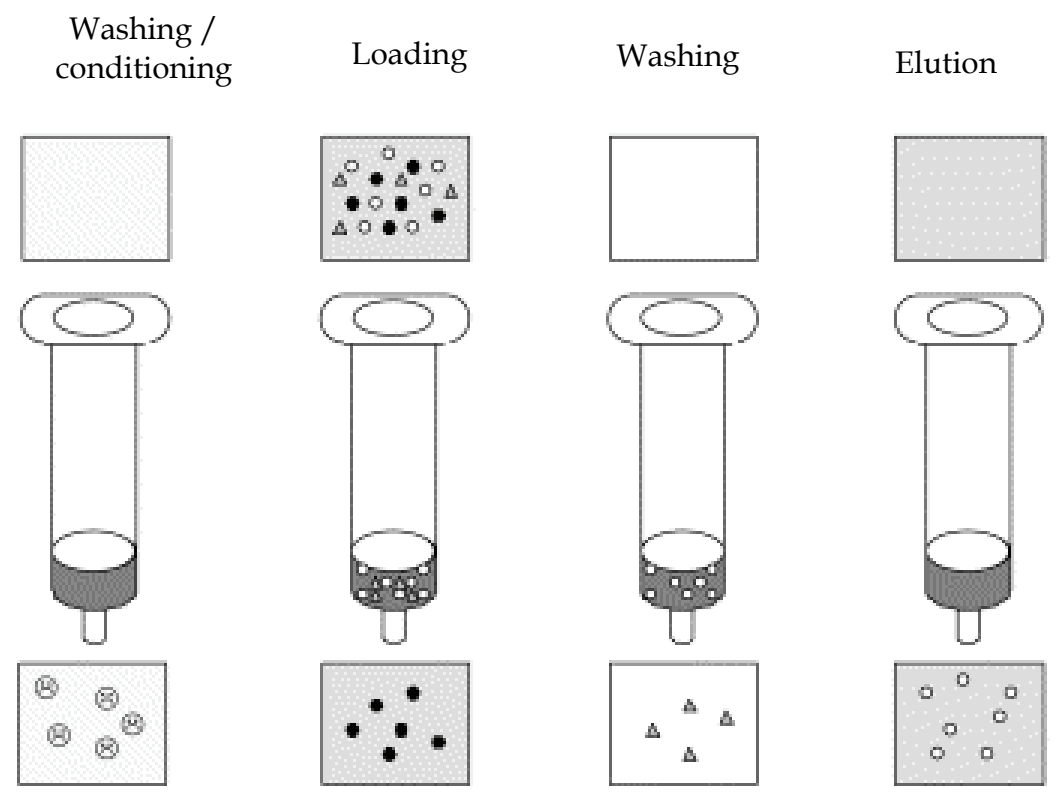

Fig. 1. SPE Operation Procedures

The final step is the elution of the analytes of interest by an appropriate solvent, without removing the retained matrix components. The solvent volume should be adjusted so that quantitative recovery of the analytes is achieved with a subsequent low dilution. In addition, the flow rate should be correctly adjusted to ensure efficient elution. It is often recommended that the solvent volume be fractionated into two aliquots, and to allow the solvent to soak into the solid sorbent before the elution.

The SPE cartridge possesses two important features, standardization and hence greater reproducibility, which includes a wide range of phases, from normal phase, reversed phase to ion-exchange materials thus enabling aqueous solutions to be treated and employing additional trapping mechanisms.

The sorbents come in different packaging: filled micro-columns, cartridge, syringe barrels and discs. The disposable sorbent containers are illustrated in Figure 2. Although the cartridges are for single use only and disposable, thus representing a significant consumable cost, this has been shown to be much lower than the cost of chemicals and the manpower needed for the corresponding traditional solvent extraction methods. Other types of SPE have also been developed, including flat disks with the stationary phase particles supported on a mesh, enabling very large volumes to be rapidly extracted. Recent use of high flow rates through extraction cartridges has been shown to give improved extraction but such "turbulent flow extractions" were very similar to conventional extractions.

Many of the published methods for pesticide determination in fresh fruits and vegetables use a combination of two or more commercially available SPE columns for cleanup in the 


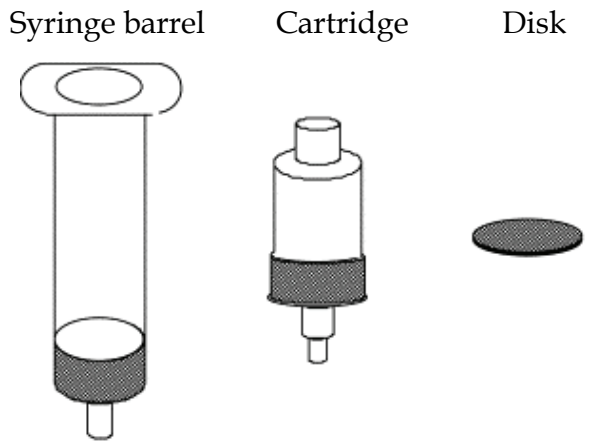

Fig. 2. Disposable SPE Sorbent Containers

normal-phase (NP) mode. Weak anion-exchange sorbents such as primary secondary amine (PSA), aminopropyl $\left(\mathrm{NH}_{2}\right)$, or diethylaminopropyl (DEA) modified silica are often used for cleanup of food samples together with strong anion-exchange sorbents (SAX) (Sharif et al., 2006). Other SPE cleanup approaches include the combination of GCB (graphitized carbon black) and PSA columns (Abhilash et al., 2007). In addition, there are other applications using reversed-phase (RP) SPE for pre-concentration / cleanup of pesticide residues from fruit and vegetable samples.

Before the SPE technique can be applied to a solid matrix such as fruits and vegetables, a separate homogenization step and often filtration, sonication, centrifugation and liquidliquid cleanup are required. Stajnbaher and Zupancic-Kralj (2003) used solid-phase extraction on a highly cross-linked polystryrene divinylbenzene column (LiChrolut EN) for the simultaneous isolation of 90 pesticides of different physico-chemical properties from fruits and vegetables and pre-concentration of the pesticides from the water-diluted acetone extract. It only used small volumes of solvent per sample $(30 \mathrm{ml}$ acetone and $14 \mathrm{ml}$ ethyl acetate, $6 \mathrm{ml}$ methanol). The majority of pesticide recoveries for various fruits and vegetables exceeded $80 \%$ in the concentration range from 0.01 to $0.50 \mathrm{mg} / \mathrm{kg}$.

Hernandez et al. (2006) determined pesticide residues in fruits and vegetables by SPE. $20 \mathrm{~g}$ of homogenized sample was mixed in $60 \mathrm{~mL}$ of methanol:water $(80: 20, \mathrm{v} / \mathrm{v})$ 0.1\% HCOOH. The mixture was extracted for $2 \mathrm{~min}$ at $8000 \mathrm{rpm}$ and filtered. The filtrate was diluted with MeOH:H2O (80:20, v/v) 0.1\% HCOOH to $100 \mathrm{~mL}$. Then, the extract was diluted eight times with LC-grade water. $2.5 \mathrm{~mL}$ aliquot was taken and diluted to $20 \mathrm{~mL} .5 \mathrm{~mL}$ of diluted extract was passed through a SPE cartridge, preconditioned with $5 \mathrm{~mL}$ of $\mathrm{MeOH}, 5 \mathrm{~mL}$ of methanol:methyl tert buthyl ether $(10: 90, \mathrm{v} / \mathrm{v}) 0.1 \% \mathrm{HCOOH}, 5 \mathrm{~mL}$ of $\mathrm{MeOH} 0.1 \% \mathrm{HCOOH}$ and finally with $5 \mathrm{~mL}$ of acidified water. The cartridge was dried for 1 hour before the sample was loaded and eluted with $5 \mathrm{~mL} \mathrm{MeOH:MTBE} \mathrm{(10:90,} \mathrm{v/v)} \mathrm{0.1 \%} \mathrm{HCOOH.} \mathrm{The}$ recoveries ranged from $70 \%$ to $110 \%$ with satisfactory precision $(<15 \%)$ that could be used for the accurate determination of 52 pesticides and metabolites in one single determination step at the $0.01 \mathrm{mg} / \mathrm{kg}$ level.

Shimelis et al. (2007) evaluated the performance of different sorbents for cleanup of oily vegetable matrices. The authors highlighted the use of a dual-layer SPE, using a combination of PSA with GCB for sample cleanup during multi-residue pesticide screening of agricultural and food products. The retention of fatty acids by the PSA sorbent was quantified and the effect of the elution solvent on the retention of fatty acid on the SPE 
cartridge was evaluated. The use of stronger elution solvents to elute certain pesticides from graphitized carbon was shown to interfere with the capacity of PSA to bind fatty acids. The ability of the PSA to retain fatty acids was found to be highly dependent on the conditioning and elution protocols. A necessity to use a stronger solvent in order to elute polar pesticides from dual-layer GCB/PSA SPE weaken the capacity of the PSA for removal of fatty acids from the samples. Therefore, practical applications of dual-layer GCB/PSA cartridges should be limited to food samples with lower levels of fatty acids.

Stajnbaher and Zupancic-Kralji (2008) determined 24 pesticides representing different chemical classess (OPPs, OCPs, carbamates and pyrethroids) in fruits and vegetables using GC-MS. The samples were homogenized and extracted by adding $18 \mathrm{~mL}$ acetone. The sample was thoroughly mixed in a vortex for $2 \mathrm{~min}$ and the extract was centrifuged for 10 $\min$ at $4000 \mathrm{rpm}$. The supernatant was transferred to a volumetric tube and acetone:water $(2: 1, \mathrm{v} / \mathrm{v})$ mixture was added. After shaking, a $2.5 \mathrm{~mL}$ aliquot corresponding to $1 \mathrm{~g}$ of sample was transferred to a $15 \mathrm{~mL}$ reservoir of a funnel shaped SPE column filled with $150 \mathrm{mg}$ of LiChrolut EN sorbent which was washed with $4 \mathrm{~mL}$ of ethyl acetate and preconditioned with $4 \mathrm{~mL}$ of methanol followed by $5 \mathrm{~mL}$ of deionized water. The pesticides retained were then eluted with $4 \mathrm{~mL}$ of ethyl acetate. Recoveries were found to be between $70 \%$ and $110 \%$ for most of the pesticides. It was reported that the miniaturized SPE method in connection with programmed temperature vaporizing (PTV)-based large volume injection was faster in sample preparation and economically beneficial as it reduced the costs of the SPE material and use of solvents.

In comparison with traditional liquid extraction techniques, SPE is simpler, more convenient, and easier to automate. In addition, SPE possesses other distinct advantages including: (1) requires a lower volume of solvent than traditional liquid-liquid extractions; (2) involves simple manipulations which are not time consuming and makes it possible for field treatment of samples; (3) the SPE cartridges can be used for short-term storage of the compounds; (4) provides high enhancement factors proportional to the volume of water passed through the SPE cartridges. One of the drawbacks of the SPE method is that the packing must be uniform to avoid poor efficiency and although the pre-packed commercial cartridges are now considered reliable, solid and oily components in a sample matrix may plug the SPE cartridge or block pores in the sorbent causing it to become overloaded and also automated systems can have difficulties with reproducibility for some sample types. The sample matrix can also affect the ability of the sorbent to extract the analyte due to competition for retention. Many traditional sorbents are limited in terms of selectivity and insufficient retention of very polar compounds can pose a problem. The use of hydrophilic materials for the improved extraction of the more polar compounds by SPE was detailed by Fontanals et al. (2005). A comprehensive review, covering trends, method development, coupled with liquid chromatography and different types of SPE sorbent materials was published by Hennion (1999) and some examples of the use of SPE in food analysis were given in a review by Buldini et al. (2002).

\section{Solid-phase Microextraction (SPME)}

Solid-phase microextraction (SPME), was developed by Pawliszyn and co-workers in 1990 in an attempt to redress the limitations inherent in SPE and LLE (Kataoka et al., 2000). It is a new sample preparation technique using a fused-silica fiber that is coated on the outside with an appropriate stationary phase. The analyte in the sample is directly extracted and 
concentrated onto the fiber coating. The method saves preparation time, solvent usage and disposal costs, and can improve the detection limits (Pawliszyn, 1997). It has been used routinely in combination with GC and HPLC, and successfully applied to a wide variety of compounds, especially for the extraction of volatile and semivolatile organic compounds from environmental, biological and food samples (Eisert and Levsen, 1996; Pawliszyn, 1997; Prosen and Zupancic-Kralj, 1999). The main advantages of SPME extraction compared to solvent extraction are the reduction in solvent use, the combination of sampling and extraction into one step and the ability to examine smaller sample sizes. It can also have high sensitivity and can be used for polar and non-polar analytes in a wide range of matrices by linking to both GC and LC.

There are currently three SPME modes that require either fused-silica fibers or GC capillary columns. Headspace (HS) and direct immersion (DI) SPME are the two fiber extraction modes, while the in-tube SPME mode is applied in the LC or HPLC instrument.

In the DI-SPME mode, the fiber is inserted into the sample medium and the analytes are transported directly to the extraction phase. For aqueous matrices, more efficient agitation techniques, such as fast sample flow, rapid fiber or vial movement, stirring or sonication are required. These actions are undertaken to reduce the effect caused by the "depletion zone" which occurs close to the fiber as a result of fluid shielding and slow diffusion of analytes in the liquid media. DI-SPME is the most common mode for pesticide analysis, and is conducted by directly inserting the fiber into the sample matrix. A method for the determination of seven OP pesticides in fruits and fruit juice samples was developed and validated by Simplicio and Boas (1999). Mean recoveries were all above $75.9 \%$ and below $102.6 \%$ for juice and between $70 \%$ and $99 \%$ for the fruit samples. Limits of detection of the method for fruits and fruit juice matrices were below $2 \mu \mathrm{g} / \mathrm{kg}$ for all pesticides. Beltran et al. (2003) has developed a DI-SPME method for the determination of seven pyrethroid pesticides in tomatoes and strawberries. Detection limits for tomato and strawberry samples were between 0.003 and $0.025 \mathrm{mg} / \mathrm{kg}$ with RSD values of less than $25 \%$. Residues of metobromuron, monolinuron and linuron herbicides and their aniline homologs in carrots, onions and potatoes have been quantified with DI-SPME with the polyacrylate (PA) fiber. A juice was obtained from samples, then diluted, added with sodium chloride and buffered. Recoveries obtained were between 76 - 95\% with RSD values of less than 10\% (Berrada et al., 2004). Sagratini et al. (2007) developed a new analysis method to detect carbamates and phenylurea pesticide residues in fruit juices using DI-SPME coupled with LC/MS and LC/QIT-MS. The pesticide residues present in watery matrices such as fruit juices were extracted using three types of fibers: $50-\mu \mathrm{m}$ Carbowax/templated resin (CW/TPR), $60-\mu \mathrm{m}$ poly(dimethylsiloxane) /divinylbenzene (PDMS/DVB) and $85-\mu \mathrm{m}$ polyacrylate. After extraction, the desorption (in a static mode) was performed in the specific interface chamber SPME/HPLC, previously filled with $70 \%$ methanol and 30\% water. The best recoveries, evaluated at two fortification levels $\left(0.2\right.$ and $\left.0.5 \mathrm{mg} \mathrm{kg}^{-1}\right)$ in fruit juices, were obtained using PDMS/DVB and CW/TPR fibers, and ranged from 25 to $82 \%$ (monolinuron, diuron and diethofencarb), with relative standard deviations (RSDs) from 1 to $17 \%$. All the limits of quantification (LOQs) were in the range of $0.005-0.05 \mu \mathrm{g} \mathrm{ml}^{-1}$ which are equal to, or lower than the maximum residue limits (MRLs) established by the Italian and Spanish legislations. A new vanguard-rearguard analytical method for determining 54 pesticide residues in different fruit juices (natural and commercial orange, peach and pineapple juices were tested) is proposed by Cortés-Aguado et al. (2008). First, pesticides are quickly extracted with ethyl acetate in a test tube, transferred to a mixture of water:acetone 9:1 (v/v), and 
isolated by solid-phase microextraction (SPME). Only $1 \mathrm{~mL}$ of juice sample is required for the analysis. The combination of a solvent and SPME extractions offer a significant selectivity and sensitivity with a proven reduction of false-positive and false-negative cases. The use of a vanguard-rearguard strategy can reduce by $50 \%$, the total time required for routine determination of juice samples in a laboratory following the accepted strategy of identification, confirmation and quantification of the pesticides in the samples by a conventional analytical method.

In the headspace sampling mode, the analyte is transported through a layer of gas before reaching the coating. This protects the fiber coating from damage by high molecular weight substances and other non-volatile concomitants present in the liquid sample matrix, such as humic materials or proteins. The amount of analyte extracted at equilibrium using DI or HS sampling are identical as long as the sample and gaseous headspace volumes are the same. This is a result of the equilibrium concentration being independent of the fiber location in the sample/headspace system. If the above condition is not satisfied, a significant sensitivity difference between the direct and headspace technique exists only for very volatile analytes. The choice of sampling mode has a significant impact on the extraction kinetics. When the fiber coating is in the headspace, the analytes are removed from the headspace first, followed by indirect extraction from the matrix. Therefore, volatile analytes are extracted faster than semivolatile components since they are at a higher concentration in the headspace, which contributes to faster mass transport rates through the headspace. The temperature has a significant effect on the kinetics of the process by determining the vapor pressure of the analytes. The equilibrium times for volatile components are shorter in the headspace SPME mode than for direct extraction under similar agitation conditions. This outcome occurs as a result of two factors: a substantial portion of the analyte is in the headspace prior to extraction, and the diffusion coefficients in the gas phase are about four orders of magnitude greater than in the liquid media. Navalon et al. (2002) determined the fungicides, pyrimethanil and kresoxim-methyl in green groceries by HS-SPME. The analysis yielded good reproducibility with the RSD values between $7.4 \%$ and $15 \%$. Lambropoulou and Albanis (2003) extracted and quantified seven OP pesticide residues in strawberries and cherries in the HS-SPME at an LOD $<13 \mu \mathrm{g} / \mathrm{kg}$. HS-SPME has been used to quantify eight pesticides in wine and fruit juices (Zambonin et al., 2004).

Chai M.K., Tan, G.H., \& Asha, L. (2008) and Chai, M.K. and Tan, G.H. (2009) optimized and evaluated the headspace solid-phase microextraction (HS-SPME) for the simultaneous determination of multiclass pesticide residues in fruits and vegetables by GC-ECD. The average recoveries obtained for each pesticide ranged between $71 \%$ and $98 \%$ at three fortification levels with the relative standard deviation of less than $5 \%$. Repeatability $(0.3-$ $3.7 \%)$ and intermediate precision (0.8-2.5\%) were shown to be satisfactory. The limits of detection $(0.01-1 \mu \mathrm{g} \mathrm{L}-1)$ and the limits of quantification $(0.05-5 \mu \mathrm{g} \mathrm{L}-1)$ of these pesticides were much lower than the maximum residue levels (MRL), allowed for fruits and vegetables in Malaysia.

In-tube SPME using an open tubular capillary column as the SPME device was developed to couple directly with an HPLC or LC-MS. It is suitable for automation, and can continuously perform extraction, desorption and injection using a standard autosampler. With the in-tube SPME technique, organic compounds in aqueous samples are directly extracted from the sample into the internally coated stationary phase of a capillary column, and then desorbed by introducing a moving stream of mobile phase or static desorption solvent when the analytes are more strongly absorbed onto the capillary coating. The capillaries selected have 
coatings similar to those of commercially available SPME fibers. The capillary column is placed between the injector loop and the injection needle of the HPLC autosampler. While the injection syringe repeatedly draws and ejects samples from the vial under computer control, the analytes partition from the sample matrix into the stationary phase until equilibrium is reached.

Subsequently, the extracted analytes are directly desorbed from the capillary coating by mobile phase flow or by aspirating a desorption solvent. The desorbed analytes are transported to the HPLC column for separation, and then detected with the UV or mass selective detection. Mitani et al. (2003) applied an automated on-line method for the determination of the isoflavones, daidzein and genistein in soybean foods by using in-tube SPME coupled to HPLC. The detection limits obtained were $0.4-0.5 \mathrm{ng} / \mathrm{mL}$ and the recoveries were above $97 \%$. Another potential advantage of in-tube SPME is that it can be easily coupled to miniaturized chromatographic systems thus enhancing the sensitivity. This has been illustrated for triazines by Chafer-Pericas et al. (2006). The limits of detections obtained for such pesticides were about 250 - 500 times lower than those achieved by using on-fibre SPME combined with conventional LC.

The fiber used in SPME is coated with a thin polymeric film, which concentrates the organic analytes during absorption or adsorption from the sample matrix. There are two mechanisms, absorption or adsorption according to the nature of the fiber. If the fiber is a liquid phase, the analyte are extracted by absorption; if the fiber is a porous particle blend, the analytes are extracted by adsorption. Absorption is a non-competitive process where analyte dissolve into the bulk of the liquid, whereas adsorption is a competitive process where analytes bind to the surface of the solid (Pawliszyn, 1999). In the adsorption case, there are a limited number of sites where analytes can bind to. When all the sites are occupied, the fiber is saturated. Therefore the linear range of the adsorption-type fibers is smaller than the one for absorption-type fibers. In a competitive process, analytes of higher affinity for the coating can displace analytes of lower affinity for the fiber. A large number of fiber coatings based on solid sorbents are now available, in addition to the original generalpurpose poly(dimethylsiloxane) (PDMS) and poly(acrilate) (PA) coated fibers, namely: PDMS/divinylbenzene (DVB), Carbowax/DVB, Carbowax/template resin (TR), Carbowax/PDMS, and DVB/Carboxen/PDMS-coated fibers. Extraction of analytes by the new porous polymer SPME fibers with mixed coating is primarily based on adsorption rather than absorption. Some of these porous polymer SPME fibers with bipolar characteristics can be very useful for the simultaneous analysis of pesticides, enlarging the spectrum of SPME applications (Cai et al., 2006).

Menezes-Filhoa et al. (2010) developed a method for the simultaneous analysis of 14 pesticide residues (clofentezine, carbofuran, diazinon, methyl parathion, malathion, fenthion, thiabendazole, imazalil, bifenthrin, permethrin, prochloraz, pyraclostrobin, difenoconazole and azoxystrobin) in mango fruit, based on SPME coupled to GC-MS. Different parameters of the method were evaluated. The best results were obtained using polyacrylate fiber and direct immersion mode at $50{ }^{\circ} \mathrm{C}$ for $30 \mathrm{~min}$, along with stirring at $250 \mathrm{rpm}$ and desorption for $5 \mathrm{~min}$ at $280^{\circ} \mathrm{C}$. The method was validated using mango samples spiked with pesticides at concentration levels ranging from 33.3 to $333.3 \mu \mathrm{gkg}^{-1}$. The average recoveries $(n=3)$ for the lowest concentration level ranged from 71.6 to $117.5 \%$, with RSD between 3.1 and $12.3 \%$, respectively. Detection and quantification limits ranged from 1.0 to $3.3 \mu \mathrm{gkg}^{-1}$ and from 3.33 to $33.33 \mu \mathrm{gkg}^{-1}$, respectively. 
Amongst the advantages it should be stated that the SPME method has a higher degree of automation when compared to other more laborious protocols. The procedure can be completely automated using an autosampler with SPME equipment. Other advantages of SPME are the inherent high sensitivity and the absence of solvents and sample pretreatment required, thus minimizing the sample manipulation and contamination. The main disadvantages include poor fiber-to-fiber reproducibility, and poor precision and ruggedness on the determination. The technique is limited to relatively semi-volatile or volatile compounds, and matrix-effects showed up in complex matrices. Finally, relatively expensive consumables and a dedicated and skilled optimization of different experimental conditions and parameters are required, making the SPME method not as straightforward for multi-residue method development.

\section{Matrix Solid-phase Dispersion (MSPD)}

Since its introduction in 1989, matrix solid phase dispersion (MSPD) has been cited as the extraction method employed in over 250 studies (Barker, 2007). It has proven to be an efficient and somewhat generic technique for the isolation of a wide range of drugs, pesticides, naturally occurring constituents and other compounds for a wide variety of complex plant and animal samples. MSPD combines aspects of several analytical techniques, performing sample disruption while dispersing the components of the sample on and into a solid support, thereby generating a chromatographic material that possesses a particular character for the extraction of compounds form the dispersed sample.

In the MSPD process, a sample (liquid, semi-solid or solid) is placed in a glass or agate mortar containing an appropriate bonded-phase or other solid support material such as octadecylsiloxane (ODS) and derivatized silica $\left(\mathrm{C}_{18}\right)$ or other suitable support materials (Figure 3).

The solid support and sample are manually blended together using a glass or agate pestle, a step that takes about 30 seconds. When blending is complete, the sample is then packed into an empty column or on top of a solid-phase extraction (SPE) sorbent without any further drying or cleanup prior to elution. The column is often an empty syringe barrel or a cartridge with a stainless-steel or polypropylene frit, cellulose filter or a plug of silanized glass wool at the bottom. A second frit or plug is often placed on top of the sample before compression with a syringe plunger. The main difference between MSPD and SPE is that the sample is dispersed throughout the column and not retained in only the first few millimeters. As regards elution, there are two possibilities: (a) the target analytes are retained on the column and interfering compounds are eluted in a washing step, followed by the target analytes being eluted by a different solvent; or (b) the interfering matrix components are selectively retained on the column and the target analytes directly eluted. Finally, additional cleanup is performed or the sample is directly analyzed. Sometimes, the MSPD column is coupled on line with an SPE column or, as in several recent applications; the SPE sorbent is packed in the bottom part of the MSPD column to remove interfering matrix components (Kristenson et al., 2006).

Several factors have been examined for their effects in the MSPD extraction. These include:

a. the effects of average particle size diameter, where as expected, very small particle sizes

(3-10 $\mu \mathrm{m}$ ) would lead to extended solvent elution times and the need for excessive pressures or vacuum to obtain an adequate flow. A blend of silicas possessing a range 
of particle sizes $(40-100 \mu \mathrm{m})$ works quite well and such materials also tend to be less expensive.

b. the character of the bonded-phase. Depending on the polarity of the phase chosen, various effects on the results may be observed. Applications requiring a lipophilic bonded-phase employ $\mathrm{C}_{18}$ and $\mathrm{C}_{8}$ materials interchangeably.

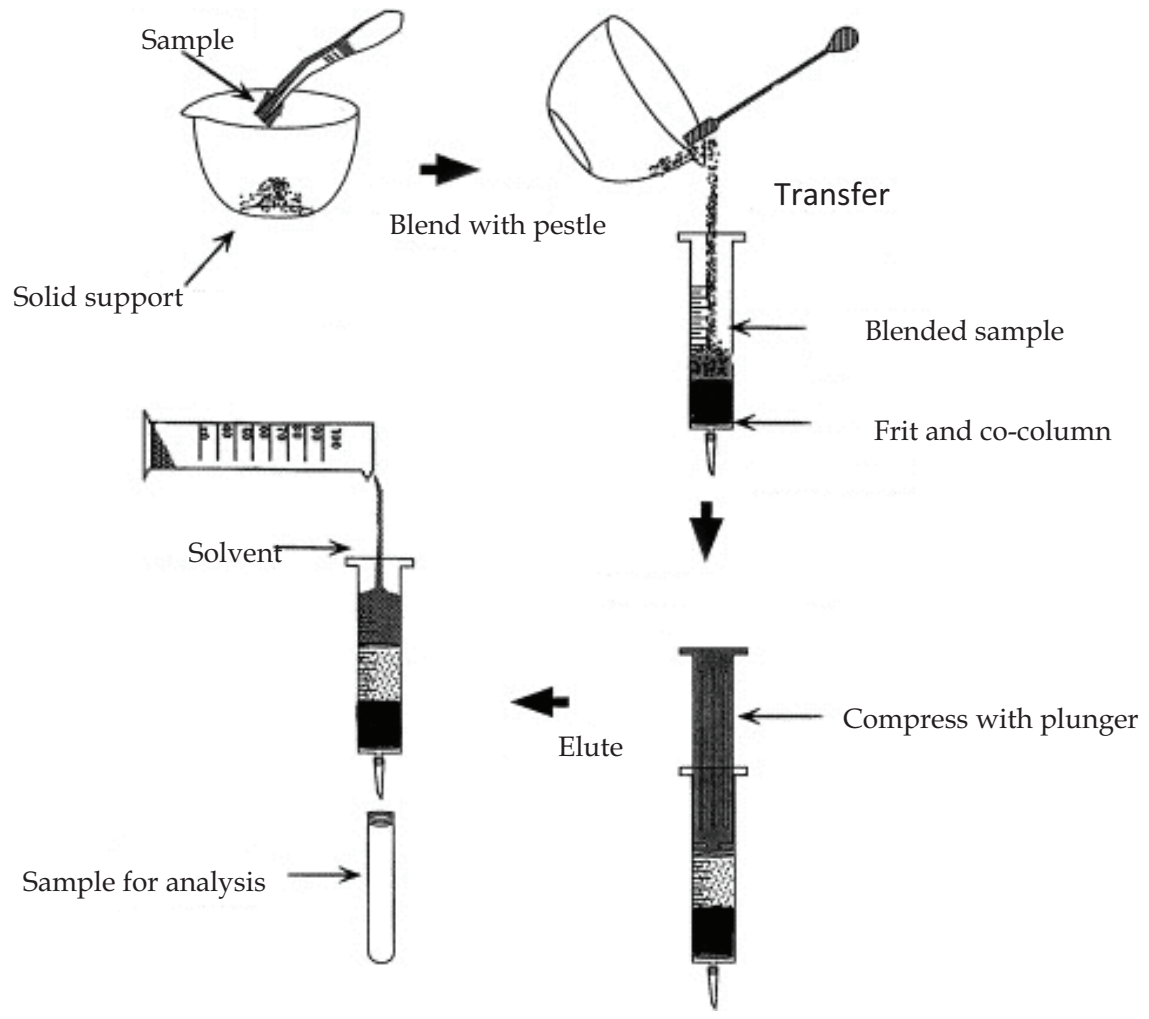

Fig. 3. MSPD Extraction Procedures (Barker, 2007)

c. the use of underivatized silica or other solid support materials. Use of unmodified or underivatized solids, such as sand to blend samples do not work in exactly the same manner as originally described for the bonded-phase solid support, such as ODS. Silicabased support materials (derivatized silica, silica gel, sand, florisil) are still being used almost exclusively in MSPD. Blasco et al. (2004) have demonstrated the use of an activated carbon fiber for the isolation of dithiocarbamates from fruits, vegetables and cereals.

d. the best proportion ratio of sample to solid support material. The most often applied is 1 to 4 , respectively, but it can vary from application to application. This ratio is dependent on the method employed. Both smaller and greater ratios have been used successfully.

e. Chemical modification of the matrix or matrix solid support blend. Addition of chelating agents such as acids and bases at the time of blending would affect the 
distribution and elution of target analytes from the sample. The solution profile of matrix components is likewise affected.

f. The optimum choice of eluent and the sequence of their application to a column. The elution solvent sequence is to isolate the analyte or further clean the column of interfering substances with each solvent step. MSPD columns permit isolation of analytes with different polarities or the entire chemical classes of compounds in a single solvent, making MSPD amenable to multiresidue analysis on a single sample. Several recent studies have reported the use of hot water as an eluting solvent as well as the addition of pressure, which is known as pressurized-liquid extraction (PLE) or accelerated solvent extraction (ASE) (Bogialli et al., 2004). Such applications demonstrate the potential to make extraction methods based on MSPD free of hazardous solvents and even less expensive to perform.

g. The elution volume. It has been observed that for an $8 \mathrm{ml}$ elution of a $2 \mathrm{~g}$ MSPD column blended with $0.5 \mathrm{~g}$ sample, the target analytes usually elute in the first $4 \mathrm{ml}$, which is approximately one column volume. This will vary for each application and should be examined to reduce the use of solvent and the unintended co-elution of potential interferences.

h. The effect of the sample matrix itself. All the components of the sample are dispersed throughout the column, covering much of the bonded-phase solid support surface, creating a new phase that can have dramatic effects on isolation in going from one matrix to another (Barker, 2000a; Barker, 2000b).

Kristenson et al. (2001) developed a miniaturized automated MSPD method for extracting pesticides from apples, pears and grapes. Only $25 \mathrm{mg}$ of sample and $0.1 \mathrm{ml}$ ethyl acetate were used and the extracts were analyzed by GC-MS without any further purification. In terms of recovery, $\mathrm{C}_{18}, \mathrm{C}_{8}$ and silica were compared for use as dispersants. The best results were obtained by using $C_{18}$. The LODs were $4-90 \mu \mathrm{g} / \mathrm{kg}$. Bogialli et al. (2004) developed a simple, rapid and specific method for analyzing seven widely used carbamate insecticides in fruits and vegetables. After matrix deposition on crystobalite (sand), the analytes were extracted with water, heated to $50-100{ }^{\circ} \mathrm{C}$. At $50{ }^{\circ} \mathrm{C}$, recoveries were between 76 to $99 \%$.

A method based on MSPD and GC was proposed for the determination of OC and pyrethroid insecticides in tea leaves ( $\mathrm{Hu}$ et al., 2005). After evaluating various extraction conditions, Hu et al. (2005) found that the best compromise in terms of recovery and cleanup was the use of Florisil as the dispersant and hexane-dichloromethane (DCM) as the extractant. LODs of the method ranged between 2 and $60 \mathrm{ng} / \mathrm{g}$, which are lower than the MRLs set by the EU. Barker et al. (2000b), Bogialli and Corcia (2007) detailed a number of applications of MSPD for the analysis of residues and Kristenson et al. (2006) detailed advances in the technique.

Recently, Cunha et al. (2007) proposed a similar approach for the determination of phosmet and its metabolites in olives using $\mathrm{C}_{18}$ and $\mathrm{MgSO}_{4}$ as sorbent and acetonitrile as eluting solvent. They found that $\mathrm{C}_{18}$ and $\mathrm{MgSO}_{4}$ as matrix sorbents have advantageous effects on extraction yields compared with polar sorbents such as silica, alumina, Florisil or aminopropyl. No additional cleanup step was proposed. A single-step extraction and purification method was developed for the separation of 26 OCPs, 3 pyrethroid pesticides and 6 PCBs from fatty foods of either animal or vegetable origin (portions of meat adipose tissues, meat products, milk and milk products, cheese, eggs, etc.) (Kodba et al., 2007). The method included homogenization of the isolated fat and DE (celite). Separation was achieved using a mini Pasteur pipette where a MSPD was carried out with only $5 \mathrm{~mL}$ of 
dimethyl sulphoxide (DMSO) as the eluting solvent. A Pasteur pipette was joined to a prepacked slurry filled Florisil column, where the LLE and adsorption chromatography successively took place. Recoveries for PCBs were from $81 \%$ to $86 \%$ and for OCPs $68-94 \%$ but one, which gave lower, and more variable recoveries. Excellent recoveries were obtained for pyrethroid pesticides, mostly more than $80 \%$. The method was applied to 509 fatty samples for monitoring these compounds.

An ultrasonic-assisted MSPD method employing $\mathrm{C}_{8}$ as sorbent was developed for extracting and cleaning-up 15 OPs and 9 triazines in fruits (Ramos et al., 2008), in order to increase process efficiency. The method performances were compared between those of a conventional MSPD with those of a heat-assisted MSPD. The employment of a sonoreactor allowed the reduction of the sonication time to 1-3 $\mathrm{min}$, and consequently overcomes the possible analyte degradation associated with increased temperatures occurring in longer sonication times. The low method detection limits(MDLs) of the ultrasound-assisted MSPD method ensured proper determination of maximum allowed residue levels for all, except for dimethoate and disulfuton. All the evaluated samples, such as apples, pears and apricots, showed a low or no matrix effect with this method.

Silve et al. (2008) proposed a simple and effective extraction method based on MSPD to determine dimethoate, malathion, lufenuron, carbofuran, 3-hydroxycarbofuran, thiabendazole, difenoconazole and trichlorfon in coconut pulp using gas-chromatographymass spectrometry. Different parameters of the method were evaluated, such as type of sorbent $\left(\mathrm{C}_{18}\right.$, alumina, silica gel and Florisil), the amount of sorbent and eluent (dichloromethane, acetone ethyl acetate, acetonitrile, n-hexane and n-hexane:ethyl acetate $(1: 1, \mathrm{v} / \mathrm{v})$. The best results were obtained using $0.5 \mathrm{~g}$ of coconut pulp, $1.0 \mathrm{~g}$ of $\mathrm{C}_{18}$ as dispersant sorbent, $1.0 \mathrm{~g}$ of Florisil as cleanup sorbent and acetonitrile saturated with nhexane as the eluting solvent.

Analysis of pesticides belonging to different classes was performed by LC-MS/MS after MSPD using diatomaceous earth as dispersant and dichloromethane as eluent (Radišić et al. 2009). Significant matrix effects observed for most of the pesticides tested were eliminated using matrix-matched calibration plots. Recoveries were in the range $71-118 \%$, with RSDs between $5-15 \%$. It was determined that the $\mathrm{pH}$ had a decisive influence on the carbendazim recovery, while its influence was not so prominent for other tested pesticides. The highest recoveries for carbendazim were obtained with the $\mathrm{pH}$-value adjusted to 6, and a slight increase in recoveries of other pesticides was also observed.

The main advantages of MSPD extraction are that besides requiring only small amounts of sample and solvents, it is rapid, inexpensive and can be carried out under mild extraction conditions (room temperature and atmospheric pressure) and provides acceptable yield and selectivity, and thus, in turn, decreases environmental contamination and improves worker safety. Moreover, the flexibility and versatility of MSPD allows the application of the process to a wide variety of analytes and biological and environmental matrices. In fact, MSPD has shown its feasibility not only for solid or semi-solid samples, but also for the viscous samples (milk, blood, etc.). For these reasons, the employment of MSPD which was first introduced in 1989, has still grown in recent years. Although useful for the analysis of trace contaminants in food, particularly as an aid or an alternative to LLE or solid phase extraction, the MSPD technique is not easily automated and could be time-consuming for a large number sample size. Although the MSPD extracts are clean enough for direct instrumental analysis, a further cleanup step is often required, particularly with fatty matrices. 


\section{Dispersive Liquid-liquid Microextraction (DLLME)}

Dispersive liquid-liquid microextraction (DLLME) is developed by Razaee et al. (2006). The method is based on the ternary component solvent system. A mixture of a water-immiscible extraction solvent dissolved in a water-miscible disperser solvent is injected rapidly into an aqueous sample. A cloudy solution consisting of fine droplets of the extraction solvent dispersed into an aqueous phase is formed. Due to the considerably large surface area between the extraction solvent and the aqueous sample, the extraction of the analytes is achieved quickly. Then centrifugation takes place, and the extraction solvent with the analytes is sedimented and analysed by an appropriate method (Berijani et al., 2006).

DLLME is a miniaturized liquid-liquid extraction (LLE) using microliter volumes of extraction solvent, which is based on the equilibrium distribution process of the target analytes between sample solution and extraction solvent. The enrichment factor and extraction recovery are calculated as follows (Berijani et al., 2006; Rezaee et al., 2006):

$$
\begin{gathered}
\mathrm{F}=\mathrm{C}_{\text {sed }} / \mathrm{C}_{\mathrm{o}} \\
\mathrm{R}=\left(\mathrm{C}_{\text {sed }} \mathrm{V}_{\text {sed }}\right) /\left(\mathrm{C}_{\mathrm{o}} \mathrm{V}_{\text {aq }}\right)
\end{gathered}
$$

Where $F, C_{\text {sed }}$ and $C_{o}$ are the enrichment factor, the analyte concentration in the sediment, and the initial concentration of analyte in the aqueous sample, respectively; $R, V_{\text {sed }}$ and $V_{\text {aq }}$ are the extraction recovery, the volume of the sediment phase, and the volume of the aqueous sample, respectively.

The extraction efficiency for the target analyte by DLLME is influenced by many factors, such as the type of extraction and dispersive solvent, and their volume, the extraction time and salt addition (Kozani et al., 2007).

The selection of an appropriate extraction solvent is a major parameter for DLLME process. The extraction solvent should satisfy two conditions: one is the higher density of the extraction solvent than that of water, which makes it possible to separate extraction solvent from aqueous phase by centrifugation; the other is the extraction capability of extraction solvent for the compounds of interest, good chromatographic behavior, and low solubility of extraction solvent in water (Kozani et al., 2007; Farahani et al., 2007). The extraction solvent volume has a large effect on the enrichment factor. With the increase of the extraction solvent volume, the final organic phase obtained by centrifugation is increased, resulting in a decrease of the concentration of the target analyte in the organic phase. Although the extraction recovery remains constant, the enrichment factor will be decreased, leading to a decrease in the sensitivity of the determination for the target compounds. Therefore, the selection of the optimal extraction solvent volume should result in both the high enrichment factor and adequate volume for the subsequent determination after centrifugation (Kozani et al., 2007; Farahani et al., 2007).

The disperser solvent is soluble in the extraction solvent and should be miscible in water, thus enabling the extraction solvent to be dispersed as fine particles in the aqueous phase to form a cloudy solution (water/disperser solvent/extraction solvent). In such a case, the surface area between extraction solvent and aqueous phase (sample) can be infinitely large, thus increasing the extraction efficiency. The disperser solvent volume directly affects the formation of the cloudy solution, the degree of the dispersion of the extraction solvent in the aqueous phase, and subsequently, the extraction efficiency (Fattahi et al., 2007). 
In DLLME, the extraction time is defined as the interval between injecting the mixture of disperser solvent and extraction solvent and centrifugation. It is was found that the extraction time has little effect on the extraction efficiency of DLLME (Farahani et al., 2007; Fattahi et al., 2007). This is because the extraction solvent can be evenly dispersed after the formation of the cloudy solution, the transition of the analyte from aqueous phase (sample) to extraction phase can be very fast, and the equilibrium state can be subsequently achieved very quickly, resulting in a very short extraction time needed for equilibrium. A short extraction time is an advantage associated with the DLLME technique (Farahani et al., 2007; Fattahi et al., 2007).

Pesticide analysis is probably the field in which DLLME has found its major applications. Different sources of water (mainly tap, river, well and lake waters) were selected as the matrix for the DLLME. In few cases, food matrices (Zhao et al., 2007; Cunha et al., 2009; Fu et al., 2009; Moinfar et al., 2009; Ravelo-Pe'rez et al., 2009a; Ravelo-Pe'rez et al., 2009b) have also been analyzed, and to a lesser extent, probably because of the complexity of the samples and also because of the need to develop a previous pretreatment procedure based mainly on solvent or water extraction.

Zhao et al. (2007) demonstrated that there were no significant differences when acetonitrile and chlorobenzene were employed as the disperser and extraction solvents, respectively, to extract six OPPs (ethoprophos, parathion methyl, fenitrothion, malathion, chlorpyrifos and profenofos) from watermelon and cucumber. In this study, sample pretreatment consisted of adding $10 \mathrm{~mL}$ of acetonitrile to $10 \mathrm{~g}$ of sample together with $4 \mathrm{~g}$ of anhydrous $\mathrm{MgSO}_{4}$ and $1 \mathrm{~g}$ of $\mathrm{NaCl}$. The mixture was then shaken in a vortex mixer and centrifuged. In the DLLME procedure, $27 \mathrm{~mL}$ of chlorobenzene (extraction solvent) were added to $1 \mathrm{~mL}$ of acetonitrile taken from the previous extraction step and the mixture was introduced into $5 \mathrm{~mL}$ of purified water. After shaking and centrifuging, the sedimented chlorobenzene phase was collected and injected into the GC-FPD system. Recoveries for each target analyte were in the range between 67 and 111\%. The RSD varied between 2 and 9\% (n = 3). LODs were found ranging from 0.010 to $0.190 \mu \mathrm{g} / \mathrm{kg}$ for all the target pesticides. Compared with the conventional sample preparation method, the proposed method has the advantage of being quick and easy to operate, and having high-enrichment factors and low consumption of organic solvents.

Fu et al. (2009) developed a DLLME method to determine carbamate (carbaryl) and organophosphorus (triazophos) pesticide residues in water and fruit juices. Using the optimum extraction conditions -extraction solvent: tetrachloroethane, $15.0 \mu \mathrm{L}$; dispersive solvent: acetonitrile, $1.0 \mathrm{~mL}$; no addition of salt and extraction time below $5 \mathrm{~s}$, the enrichment factors for the carbaryl and triazophos were 87.3 and 275.6, respectively. The linearity was obtained in the concentration range of $0.1-1000 \mathrm{ngmL}^{-1}$ with correlation coefficients from 0.9991 to 0.9999 . The LODs, ranged from 12.3 to $16.0 \mathrm{pg} \mathrm{mL}^{-1}$. The RSDs, for $10 \mathrm{ng} \mathrm{mL}-1$ of carbaryl and $20 \mathrm{ng} \mathrm{mL}^{-1}$ of triazophos varied from $1.38 \%$ to $2.74 \%(\mathrm{n}=6)$. The relative recoveries of fruit juice samples were in the range of $86.3-105.3 \%$.

In the past decade, a new type of solvent, ionic liquids (IL), has been introduced into analytical chemistry as an extractant (Poole and Poole, 2010). ILs are low-melting salts that form liquids composed entirely of ions, which have generally been found to be less toxic, less volatile and less contaminating than conventional solvents. These salts have also been used as extraction solvents in DLLME for extracting pesticides in fruit samples (RaveloPe'rez et al. 2009a; Ravelo-Pe'rez et al. 2009b) 
The first application of IL-DLLME for the extraction of pesticides from matrices other than water was recently developed by Ravelo-Pe'rez et al. (2009a, 2009b) for the extraction of fruit extracts. In these cases, disperser solvents were used together with the IL. In the first of these studies (Ravelo-Pe'rez et al. 2009a), parameters affecting the IL-DLLME of eight pesticides (i.e. thiophanate-methyl, carbofuran, carbaryl, tebuconazole, iprodione, oxyfluorfen, hexythiazox and fenazaquin) were optimized by means of an experimental design (central composite design). The selected parameters were sample $\mathrm{pH}, \mathrm{NaCl}$ percentage, IL amount (1-hexyl-3-methylimidazolium hexafluorophosphate)- [HMIm] $\left[\mathrm{PF}_{6}\right]$ ) and methanol volume (disperser solvent). The final procedure in this study consisted of the ultrasonic- assisted extraction of $1 \mathrm{~g}$ of homogenized bananas with acetonitrile using different salts $\left(\mathrm{MgSO}_{4}, \mathrm{NaCl}\right.$, sodium hydrogen citrate sesquihydrate and sodium citrate tribasic dihydrate) to improve the recoveries. After centrifugation and evaporation of the supernatant, reconstitution of the extract in water at $\mathrm{pH} 2.7$ provided the best media to develop the IL-DLLME procedure, which used $88 \mathrm{mg}$ of [HMIm][PF 6$], 714 \mathrm{~L}$ of methanol and $28.9 \%(\mathrm{w} / \mathrm{v})$ of $\mathrm{NaCl}$. The combination of these two procedures (acetonitrile extraction of the fruit and reconstitution of the evaporated extract in water) provided a suitable arrangement that allowed application of DLLME as part of the sample-pretreatment procedure for extraction of pesticides from complex samples. Mean recovery percentages were $53-97 \%$ with RSD values below $8.7 \%$, which represent LODs of $0.320-4.66 \mu \mathrm{g} / \mathrm{kg}$, well below maximum residue limits of the European Union (EU MRLs). In the later study Ravelo-Pe'rez et al. (2009b) extended the application of the method for the extraction of the same group of pesticides from grapes and plums. Mean recovery values were $72-100 \%$ for table grapes and $66-105 \%$ for plums, with LODs very similar to those reported in the earlier study, which are aslo below the EU MRLs. In both these studies, the method was also applied to the analysis of commercial fruit samples, in which residues of pesticides could be found in some samples, with some exceeding the MRLs.

Compared to other techniques, DLLME is characterized by very short extraction times, mainly because of the large surface area between the solvent and the aqueous phase. Other advantages are simplicity of operation, low cost, and high recovery and enrichment factors, offering potential for ultra-trace analysis. However, the main drawback of DLLME is that its efficiency is restricted by solvent selection to systems capable of forming a dispersive phase, somewhat limiting its range of application by sample. However, the introduction of new solvents (e.g., ILs, which are numerous) may provide a new alternative. Automation of DLLME seems to be very difficult and has not yet been achieved, although an attempt was made for the analysis of inorganic species (Anthemidis and Ioannou, 2009).

\section{Stir-bar Sorptive Extraction (SBSE)}

Stir bar sorptive extraction (SBSE) was developed by Baltussen et al. (1999) to overcome the limited extraction capacity of SPME fibers. A glass stirrer bar is coated with a potentially thick bonded absorbent layer (polydimethylsiloxane - PDMS) to give a large surface area of stationary phase, leading to a higher phase ratio and hence a better recovery and sample capacity (Figure 4). The advantages of sorptive extraction using PDMS include predictable enrichment, the absence of displacement effects, inertness, and rapid thermal desorption at mild temperature. Stir bar sorptive extraction of a liquid sample is performed by placing a suitable amount of sample in a headspace vial. The stir bar is added and the sample is stirred, typically for $30-240 \mathrm{~min}$. the extraction time is controlled kinetically, determined by 
sample volume, stirring speed, and stir bar dimensions and must be optimized for a given application.

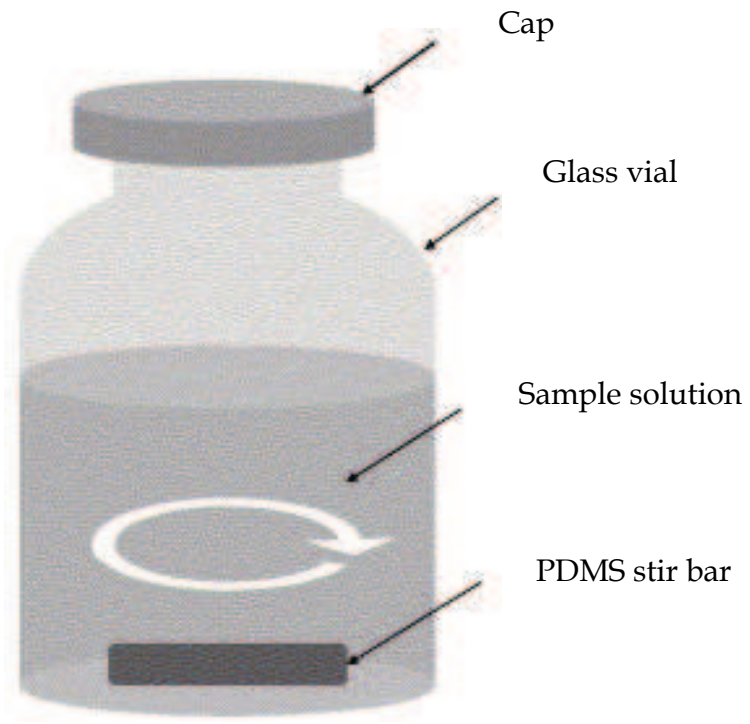

Fig. 4. Schematic Diagram of a SBSE Setup

Normally, SBSE is applied to the extraction of aqueous samples containing low concentrations of organic compounds. For samples containing high concentrations of solvents, the solutions should be diluted before extraction. For the extraction of highly nonpolar solutes, an organic modifier is added to minimize wall adsorption. Thus, the optimization of the organic modifier concentration is necessary.

After extraction, the stir bar is removed, then placed on a clean tissue paper, rinsed with distilled water to remove water droplets, and introduced in a thermal desorption unit. This step will avoid the formation of non-volatile material during the thermal desorption step. Rinsing would not cause any solute loss, because the adsorbed solutes are present inside the PDMS phase. After thermal desorption, the stir bars can be reused. Typically, the lifetime of a single stir bar is approximately 20 to 50 extractions, depending on the matrix (David and Sandra, 2007).

Since SBSE using PDMS coating is similar to liquid-liquid extraction using a non-polar solvent, the technique is mainly used for non-fatty matrices $(<3 \%$ fat). The analysis of pesticides in fruits and vegetables (Sandra et al., 2003; Juan-Garcia et al., 2004; Juan-Garcia et al., 2005; Zuin et al., 2006) has been described. After homogenization, the fruit and vegetable samples are extracted using a water miscible solvent. An aliquot of the extract is diluted with water and followed by SBSE. Both LC-MS desorption and thermal desorption GC-MS have been used.

Sandra et al. (2003) used SBSE with thermal desorption capillary GC-MS for the screening of pesticides (OPPs and OCPs) in fruits, vegetables and baby food. A $10 \mathrm{~mm}$ stir bar coated with $0.5 \mathrm{~mm}$ PDMS was used. The recoveries for spiked samples ranged between $43-75 \%$. The coupling of SBSE with RTL-GC-MS operated in the scan mode could monitor simultaneously about 300 pesticides present in fruits, vegetables and baby food. The detection limits from $\mathrm{mg} / \mathrm{kg}$ to the sub $\mu \mathrm{g} / \mathrm{kg}$ level were obtained. 
Juan-Garcia et al. (2004) studied the detection of fungicide residues in grapes by LC-MS. Two procedures based on SPE and SBSE have been assessed for extracting these compounds in grapes. The recoveries obtained by SPE in samples spiked at the LOQ level ranged from 60 to $100 \%$ with RSDs from 7 to $17 \%$. With the SBSE the recoveries obtained from samples spiked at the LOQ level were between 15 and 100\% and the RSDs between 10 and 19\%. The LOQs of most compounds are better via the SPE $\left(0.003-0.01 \mathrm{mg} \mathrm{kg}^{-1}\right)$ than by SBSE $(0.01 \mathrm{mg}$ $\mathrm{kg}^{-1}$ for all fungicides).

Guan et al. (2008) prepared a novel poly(phthalazine ether sulfone ketone) (PPESK) film and coated this on stir bars with a thickness of $250 \mu \mathrm{m}$ for sorptive extraction. The PPESK coated stir bar has high thermostability $\left(290{ }^{\circ} \mathrm{C}\right)$ and a long lifetime (50 repeated use). The extraction properties of this stir bar were evaluated for the extraction of both polar and semi-polar analytes, including organochlorine compounds and organophosphorus pesticides. The PPESK stir bar showed higher affinity towards polar compounds than that of a PDMS coated stir bar and a higher sample load compared with the corresponding PPESK fiber. It was applied to the determination of organophosphorus pesticides in juices by gas chromatographic analysis. Limits of detection for organophosphorus pesticides were in the range of $0.17-2.25 \mathrm{ng} \mathrm{L}^{-1}$ and $2.47-10.3 \mathrm{ng} \mathrm{L}^{-1}$ in grape and peach juice, respectively, using the flame thermionic detector (FTD), with precisions of less than 20\% RSD.

Although SBSE is widely applied in environmental and food analysis, it has also some limitations or drawbacks. One of the drawbacks is related to the fact that the coated stir-bar cannot be directly desorbed in a simple split/splitless injection port of a gas chromatograph. Hence the analyte has to be back extracted into a suitable solvent, which adds an additional step to the overall analytical method, or a specially designed Thermal Desorption Unit (TDU) has to be used. Moreover, operations like removing the stir-bar from the sample, rinsing and drying are usually performed manually, which is laborious and can introduce errors. Automation of these steps is possible but this increases the cost and complexity of the hardware involved. However, the most important limitations of SBSE are related to the coating of stir-bars. The non polar PDMS is at present the only polymer commercially available as a coating for stir-bars. Recovery of polar analytes is poor and often in situ derivatisation is applied to increase extraction yields. Stir-bars coated with materials with better affinity to polar compounds would improve SBSE flexibility and selectivity while maintaining its concentration capability. New approaches or concentrating materials are therefore required to overcome the above-mentioned limitation and to extend the range of applications. Up to now, developments of novel stir-bars have been reported with limited references. One of the methods developed was to use dual-phase-coated stir-bars, which combine two or more sampling materials with different concentration capabilities (Bicchi et al. 2005). These new stir-bars consist of a short PDMS tube at both ends with two magnetic stoppers, whose inner cavity is packed with different types of adsorbents such as activated carbon. Dual-phase stir-bars with carbon have been shown to improve the recovery of volatile and polar compounds compared to the conventional PDMS stir-bar.

\section{Single-drop Microextraction (SDME)}

Recently, alternative but SPME related concepts have been introduced for sample extraction. The use of a single droplet for extraction purposes was first recommended in the mid-1990s (Mester and Sturgeon, 2005). Figure 5 shows one possible embodiment of the SDME technique employing a microsyringe. The syringe needle is used to pierce the septum of a 
closed container. When the tip of the needle is in the desired position (in the aqueous phase or in the headspace) a hanging droplet of solvent is exposed to the matrix by depressing the plunger of the syringe. After extraction is completed, the droplet is withdrawn into the syringe barrel by lifting the plunger. The extracted samples can then be submitted directly to GC analysis. Thus the system requires two discrete parts: the first for extraction and the second for injection.

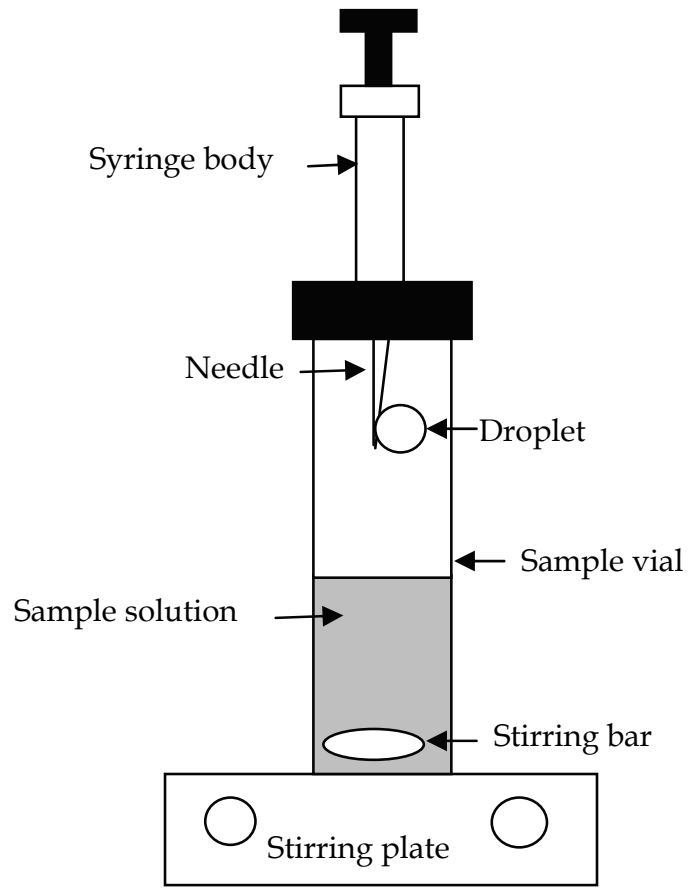

Fig. 5. Schematic Diagram of a SDME Setup (Mester and Sturgeon, 2005)

The properties of the analyte and its matrix will determine whether direct immersion (DISDME) or headspace (HS-SDME) extraction is appropriate. Thus, one must consider the volatility (boiling point), ionization (for acids and bases) and polarity of the analyte and matrix. HS-SDME is appropriate for most polar and non-polar, lower molecular weight, volatile and semivolatile compounds. DI-SDME extraction is appropriate for non-polar or moderately polar higher molecular weight, semivolatile chemicals. There are some important restrictions on the selection of a particular extracting solvent. When extracting from an aqueous solution, the solvent needs to be water immiscible. The solvent needs to have a boiling point high enough that it will not evaporate, but also appropriate for the chromatographic system. It needs to have a high enough viscosity to cling onto the tip of a syringe needle, but not so viscous that the diffusion rate of the analyte into the drop affects extraction time significantly. The intermolecular attraction characteristics of the solvent must also be compatible with the analyte being extracted. Toluene appears to be the most commonly used acceptor phase, because it has high solubility for the target analytes, is immiscible in water and stable enough over the extraction time. Based on this solvent as the 
acceptor phase, several methods were validated and applied to the determination of OP and OC pesticides in solid samples (Xiao et al., 2006; Zhao, E. et al., 2006b). Carbon tetrachloride has also been successfully applied to the extraction of OP pesticides (Ahmadi et al., 2006); this solvent is, however, more prone to dissolve or become dislodged when long extraction periods are used. Isooctane and n-hexane have been also used for the determination of OP and OC pesticides (Zhao, L. and Lee, 2001; Lopez-Blanco et al., 2003). In the SDME procedure, solvent volumes lower than $3 \mu \mathrm{L}$ are commonly used, due to the instability of the microdrop at higher values as well as to the good compatibility with the GC instruments.

SDME involves dynamic partitioning of the target compounds between the acceptor phase and the sample solution, and the extraction efficiency depends on the mass transfer of analyte from the aqueous phase to the organic solvent phase. Since the mass transfer is a time-dependent process, a graph representing the relationship between peak area and extraction time is typically reported. Generally, extraction yield increases over relatively long exposure times. Since SDME is not an exhaustive extraction technique, it is not always practical to match extraction time with extraction equilibrium, because the potential for solvent loss due to dissolution increases with time. Therefore, extraction times are rarely set at equilibrium but rather at a point where sensitivity and precision are maximized over an acceptable experimental time. For pesticide analysis, extraction times of 15-30 min are usually selected.

Agitation is a critical parameter in SDME procedures. The mass transfer of the target compounds to the organic solvent can be enhanced by agitation of the sample solution, thereby reducing the time required to attain thermodynamic equilibrium. However, excessive agitation could result in a dislodgement of the acceptor phase and difficulties in analyte quantification, especially with prolonged exposure times.

The "salting out" effect was studied, and the results showed that high salt concentrations in the aqueous samples usually decrease the diffusion of analytes toward the organic phase thus impairing the extraction. This effect is more pronounced in the case of SDME and thus most of the studies have been performed without or with a small amount of salt addition (Zhao, L. and Lee, 2001; Xiao et al., 2006; Ahmadi et al., 2006). Caution should be taken when high salt concentrations are used in the sample matrix, since under these conditions, in combination with the agitation of the sample, the formation of air bubbles was promoted, increasing the incidence of drop loss or dislodgement of organic solvent.

Generally, the studies of SDME in the determination of pesticides in food samples are very limited because of their complex matrices (Xiao et al., 2006; Zhao, E. et al., 2006b; Zhang et al., 2008; Amvrazia \& Tsiropoulos, 2009a).

Xiao et al., 2006 developed a single-drop microextraction (SDME) procedure for the analysis of organophosphorus pesticides (OPPs) in fruit juice by gas chromatography (GC) with flame photometric detection (GC-FPD). Two types of SDME mode, static and cycle-flow SDME, were evaluated. The enrichment factors for six OPPs in static SDME were nearly 100fold (except for dichlorvos 23-fold), which were much better than that in cycle-flow SDME. Therefore, static SDME with tributyl phosphate (TBP) as the internal standard was selected for the real sample analysis. A 100-fold dilution of fruit juice samples is adequate to determine levels of most pesticides below the MRLs because of the low limits of detection of the method. The recoveries for the spiked juice samples were from 77.7 to $113.6 \%$.

An approach for the extraction of 9 kinds of organochlorine pesticides (OCPs) from vegetable samples (cabbage, cauliflower, Chinese cabbage) coupling single-drop microextraction with gas chromatography-mass spectrometry was presented by Zhang et al. 
(2008). An effective extraction was achieved by suspending a $1.00 \mu \mathrm{L}$ mixed drop of $\mathrm{p}$ xylene and acetone $(8: 2, \mathrm{v} / \mathrm{v})$ to the tip of a microsyringe immersed in a $2 \mathrm{~mL}$ donor aqueous solution and stirred at $400 \mathrm{rpm}$. The relative recoveries were from 63.3 to $100 \%$, with repeatability ranging from 8.74 to $18.9 \%$ (R.S.D.). In contrast to some common acceptor solvents, a novel combination of liquids comprising p-xylene and acetone showed better extractions and lower detection limits $\left(0.05 \mathrm{ng} \mathrm{mL}^{-1)}\right.$ for organochlorine pesticides.

Amvrazia \& Tsiropoulos (2009a) evaluated the single-drop microextraction (SDME) technique coupled with GC-NPD and GC-ECD for the determination of14 types of multiclass pesticides in vegetables (tomato and courgette). The optimum sample preparation was achieved with the use of a mixture of acetone $/ \mathrm{H}_{2} \mathrm{O}(10 / 90, \mathrm{v} / \mathrm{v})$ in a donor sample solution and subsequent SDME using a toluene drop $(1.6 \mu \mathrm{L})$ under mild stirring for $25 \mathrm{~min}$. The efficiency of the extraction process was studied in fortified tomato and courgette samples and the matrix effect assessment performed showed that quantification should be performed using a standard curve of spiked vegetable samples since certain matrix components as observed in the tomato analysis, may enhance pesticide recoveries via SDME. The proposed method showed good linearity, limits of detection at the sub- $\mu \mathrm{g} \mathrm{kg}^{-1}$ level and high precision (RSD $<15 \%$ ) and was applied successfuly in real vegetable samples showing that SDME can be a promising way for sample preparation in pesticide residue analysis.

Due to its simplicity, ease of implementation, and insignificant startup cost, SDME is accessible to virtually all laboratories. However, it has some limitations, for example: (a) in its most basic form, direct immersion mode it requires careful and elaborate manual operation because of the problem of drop dislodgment and instability; (b) the SDME is affected by the presence of humic acids or suspended solids indicating that it has a limited advantage in complex matrices, in which extra filtration of the sample is necessary; (c) notwithstanding the acceptable analytical performance mentioned above, the sensitivity and the precision of SDME methods can be further improved. The main issue lies with the adverse consequences of prolonged extraction time and fast stirring rates, since they may result in drop dissolution and dislodgement; (d) SDME is not yet suitable as a routine online pre-concentration procedure. Although some progress has been made to automate SDME, cost considerations will mean that the approach will not be widely accessible (Xu et al., 2007).

\section{Pressurized Fluid Extraction (PFE)}

This technique, also named accelerated solvent extraction (ASE) or pressurized liquid extraction (PLE), is a solid-liquid extraction process performed in closed vessels at relatively elevated temperature, usually 80 to $200{ }^{\circ} \mathrm{C}$, and elevated pressures, between 10 and $20 \mathrm{MPa}$ conditions for short time periods (5-10 min). Therefore, PFE is quite similar to supercritical fluid extraction(SFE) but $\mathrm{CO}_{2}$ is replaced by organic solvents to mitigate potential polarity problems. Extraction is carried out under pressure to maintain the conventional organic solvents in its liquid state, but extracting at temperatures well above their atmospheric boiling points. Therefore, the solvent is still below its critical condition during PFE but has enhanced solvation power and low viscosities and hence allows higher diffusion rates for the analytes. In this way the extraction efficiency increases, minimizing the amount of solvent needed and expediting the extraction process. The time required for extraction is independent of the sample mass and the efficiency of extraction is mainly dependent on the temperature. Figure 6 shows a schematic diagram of a PFE system. 


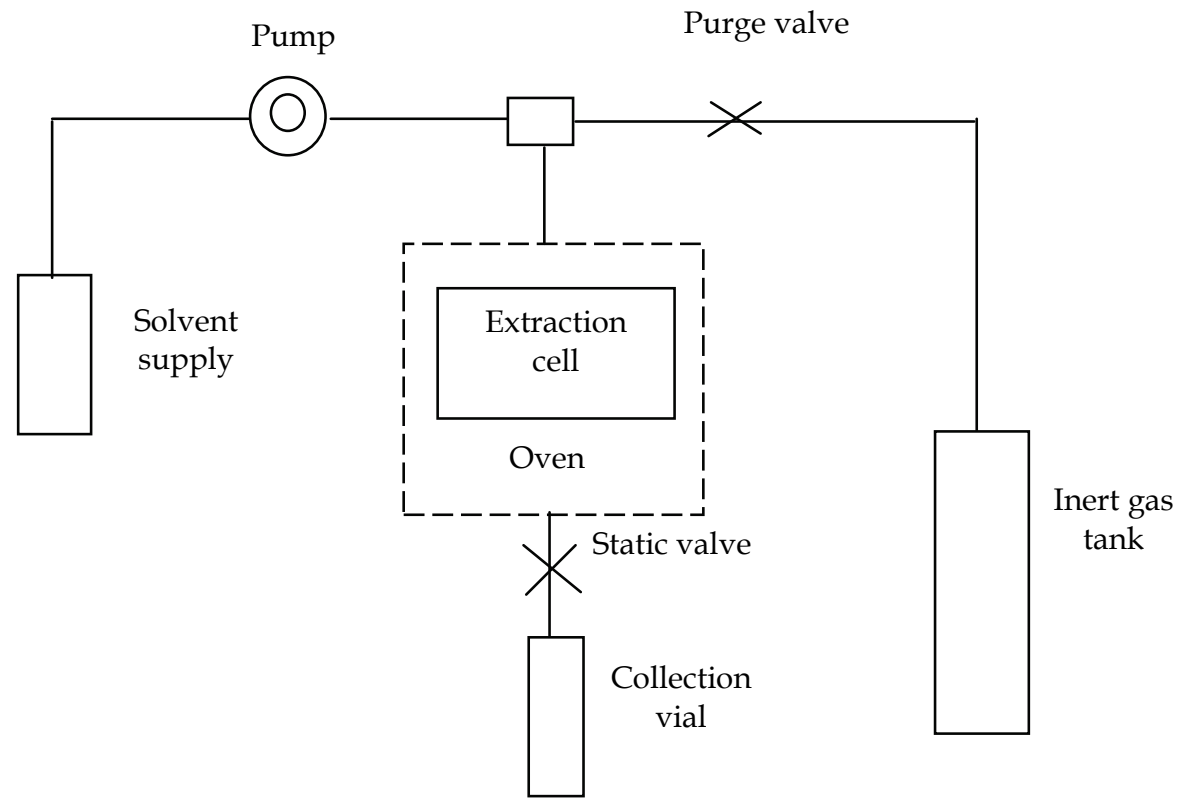

Fig. 6. Schematic Diagram of a PFE System (Buldini et al., 2002)

Both static and flow through extraction systems can be used. In the static extraction mode, the sample is loaded in an inert cell and pressurized with a solvent heated above its boiling point for some time. The extract is then automatically removed and transferred to a vial. In the flow through extraction mode, a fresh solvent is continuously introduced to the sample. This improves the extraction efficiency but, the extract is subsequently diluted. The extract is pushed into the collection vial by a second aliquot of solvent inserted into the extraction cell and this second aliquot is then collected into the same vial by pushing it with an inert gas flow. The whole process takes approximately 15-20 min.

In PFE, the pressure is applied to maintain the solvent in its liquid state. This reduces the number of parameters that need to be optimized to achieve efficient extractions compared with SFE. The main parameters to consider now are temperature and time and this reduces the time devoted to method development and optimization of the extraction procedure. The method set up is generally straightforward because the same solvent recommended in the official and routine Soxhlet methods can be used. Therefore, PFE is an attractive technique because it is fast (e.g. extraction time approximately $15 \mathrm{~min}$ per sample), uses less solvent volume $(15-40 \mathrm{ml})$, no filtration is required after extraction, the instrumentation allows extraction in unattended operation and different sample sizes can be accommodated. The two main disadvantages of PFE include limited selectivity because it usually requires further cleanup of the extract obtained and higher initial cost than SFE or microwaveassisted extraction (MAE) systems.

Tao et al. (2004) applied PFE for extracting DDT and its metabolites from wheat with hexane/acetone $(1: 1, \mathrm{v} / \mathrm{v})$ at $120{ }^{\circ} \mathrm{C}$ and a pressure of $101 \mathrm{MPa}$. Moreno et al. (2006) investigated the extraction of 65 pesticides including OC pesticides from greasy vegetable 
matrices such as avocado using PFE with ethyl acetate/cyclohexane $(1: 1, \mathrm{v} / \mathrm{v})$ at $120{ }^{\circ} \mathrm{C}$ and a pressure of $12 \mathrm{MPa}$.

Chuang et al. (2001) investigated the use of PFE for the analysis of pesticides in baby food but observed matrix interferences due to the high level of fat present in the samples. Although widely used as an initial extraction for solid samples, for trace analysis, postextraction procedures for analyte enrichment/concentration are often required. SPE can be coupled to the extractor outlet to enable cleanup and concentration. Coupling of PFE to other cleanup steps was used for the determination of pesticides in foods (Herrera et al. 2002).

When water is employed as the extraction solvent in PFE, a different terminology is used to highlight the fact that water is an environmental-friendly solvent. Thus, terms such as pressurized hot water extraction, subcritical water extraction (SWE), superheated water extraction and high temperature water extraction can be found in the literature (Ramos et al., 2002; Smith, 2003; Carabias-Martinez et al., 2005). Because the polarity of water decreases markedly as the temperature is increased, superheated water at $100-200{ }^{\circ} \mathrm{C}$, under a relatively low pressure can act as a medium to non-polar solvent (ethanol or acetone) and is an efficient extraction solvent for many analytes (Ramos et al., 2002; Smith, 2003; CarabiasMartinez et al., 2005). A review of the technique, including several applications was given by Smith in 2002.

A limitation in extracting with hot water is the inability to recover compounds that are hydrophobic, thermally labile, or easily hydrolyzed. Besides, one of the disadvantages of SWE, particularly for trace analysis is that the extract obtained is a dilute aqueous solution. This means that a further concentration / extraction step is often required prior to analysis (such as liquid-liquid extraction or solid-phase extraction). To avoid the additional cleanup, a trapping agent can be added to the extraction vessel, such as an SPE disc, which is then subsequently extracted. Commercial PLE systems can be used and it is possible to link SWE to LC, by trapping analytes onto a cartridge, prior to elution with the mobile phase. Phase transfer catalysis can be used to enable in situ derivatisation and concentration of the product into an organic solvent (Chienthavorn et al. 2006)

\section{Microwave-assisted Extraction (MAE)}

MAE uses microwave radiation $(0.3-300 \mathrm{GHz})$ as the source of heating a solid-solvent sample mixture. Due to the particular effects of microwaves on the matter namely, dipole rotation and ionic conductance, heating with microwaves is instantaneous and occurs in the bulk of the sample, leading to very fast extraction. The heat generated in the sample by the microwave field requires the presence of a dielectric compound. The greater the dielectric constant, the more thermal energy is released and the more rapid would be the heating for a given frequency. Consequently, the effect of microwave energy is strongly dependent on the nature of both the solvent and the solid matrix. Usually, the extraction solvent has a high dielectric constant, so that it strongly absorbs the microwave energy. However, in some cases especially for thermo labile compounds, the microwave may be absorbed only by the matrix, resulting in heating of the sample and the release of the solutes into the cold solvent. Therefore, the nature of the solvent is of great importance in MAE: it should selectively and efficiently solubilize the analytes in the sample but, at the same time, it should absorb the microwave without leading to a strong heating to avoid eventual degradation of the analyte compounds. Thus, it is common practice to use a binary solvent mixture (e.g. hexane- 
acetone, 1:1) where only one of the solvents is absorbing the microwave energy. Other important parameters affecting the extraction process are the applied power, the temperature and the extraction time. Moreover, the water content of the sample needs to be carefully controlled to avoid excessive heating, thus allowing reproducible results.

The application of microwave energy to the samples may be performed either in closed vessels with pressure and temperature control (pressurized MAE) or in open vessels at atmospheric pressure (focused MAE). In focused MAE method, the temperature is limited by the boiling point of the solvent at atmospheric pressure, but in pressurized MAE the temperature may be elevated by applying an adequate pressure (Dean. 2000).

The technique has proven to be better than soxhlet extraction by reducing the solvent consumption and extraction time (Diagne et al., 2002; Barriada-Pereira et al., 2003). Usually sample sizes range from 0.5 to $10 \mathrm{~g}$ and $10 \mathrm{ml}$ of solvent is sufficient for the extraction time from less than 1 to $10 \mathrm{~min}$. The same laboratory microwave unit previously described for sample digestion is used, so reducing costs; the simultaneous extraction of many different samples is also possible without any mutual interference.

Cai et al. (2003) used MAE to extract OC pesticides from Chinese teas before solid-phase microextraction followed by GC-ECD analysis. The recoveries of MAE were compared with those of ultrasonic extraction and the results showed that MAE provided better recoveries (efficiencies) and shorter extraction times than ultrasonic extraction.

Recently, MAE has been proposed for the extraction of pesticide residues in avocado, avocado oil and olive oil (Fuentes et al. 2008, Hernandez-Borges et al. 2008). Fuentes et al. (2008) proposed a method using MAE to assist the liquid-liquid extraction of pesticide in avocado oil and olive oil. An additional cleanup step using an Envi-carb SPE cartridge was proposed. The method is relatively simple, low solvent consuming and has a good throughput of samples (10 samples can be analyzed in $4 \mathrm{hrs}$ ). Hernandez-Borges et al. (2008) proposed a MAE of abamectin residues in avocado. Homogenized avocado samples were extracted once with 20 $\mathrm{mL}$ acetonitrile:water $4: 1(\mathrm{v} / \mathrm{v})$ in a microwave oven for $26 \mathrm{~min}$ at $700 \mathrm{~W}$ with a maximum temperature of $80^{\circ} \mathrm{C}$. An additional cleanup step was performed using a $\mathrm{C}_{18}$ SPE cartridge.

MAE also limits contamination or absorption from the vessel, due to direct heating of the sample. The main advantages of microwave pre-treatment are the low temperature requirement, high extraction rate, complete automation and the possibility of simultaneously extracting many different samples at the same time with little interference. However, MAE has also several drawbacks such as the extract must be filtered after extraction, polar solvents are needed, cleanup of extracts may be necessary and the equipment is moderately expensive.

\section{Supercritical Fluid Extraction (SFE)}

A general trend in the isolation of pesticide residues is to decrease the consumption of expensive and toxic organic solvents and to increase the availability of a broad range of analytes and matrices. A possible solution is to use supercritical fluid extraction (SFE). SFE uses liquids such as compressed carbon dioxide $\left(\mathrm{CO}_{2}\right)$ as an extracting phase that is capable of removing less volatile compounds at ambient temperature. Supercritical fluids possess both gas-like mass transfer and liquid-like solvating characteristics.

SFE utilizes commercially available equipment where the fluid is pumped, at a pressure above its critical point $\left(7.38 \mathrm{mPa} \& 31.1^{\circ} \mathrm{C}\right)$, with the sample placed in an inert extraction cell. The temperature of the cell is increased to overcome the critical point of the fluid. After 
depressurization, the analytes are collected in a small volume of organic solvent or on a solid-phase filled cartridge (solid adsorbent trap). Extraction can be performed in the static, dynamic or recirculating mode: in the static extraction mode, the cell containing the sample is filled with the supercritical fluid, pressurized and allowed to equilibrate; using the dynamic mode, the supercritical fluid is passed through the extraction cell continuously; finally in the recirculating mode the same fluid is repeatedly pumped through the sample and, after the required number of cycles, it is pumped out to the collection system (Figure 7). One of the most interesting properties of these fluids is the direct relationship of solvent strength to density. Since the density of the fluid is a function of its temperature and pressure, precise control of these parameters allows a solvent with a narrow window of solvating strength to be obtained. It is possible, therefore, to substitute a variety of conventional solvents with a single supercritical fluid. For instance, supercritical carbon dioxide at $7.515 \mathrm{MPa}$ and $80 \circ \mathrm{C}(\mathrm{d} 0.15 \mathrm{~g} / \mathrm{ml})$ is characterized by a solvating strength similar to gases, such as pentane, while at $38.265 \mathrm{MPa}$ and $40 \circ \mathrm{C}(\mathrm{d} 0.95 \mathrm{~g} / \mathrm{ml})$ its solvating strength resembles liquids, such as dichloromethane, carbon tetrachloride, toluene or benzene. When selecting the extraction pressure, it should be kept in mind that as the pressure increases, higher molecular weight compounds become soluble, while as the pressure decreases, the supercritical fluid loses some of its solvent strength. If the pressure is reduced to atmospheric values, the fluid loses practically all of its solvating ability and the extracted compounds fall out of the solution (Buldine et al., 2002).

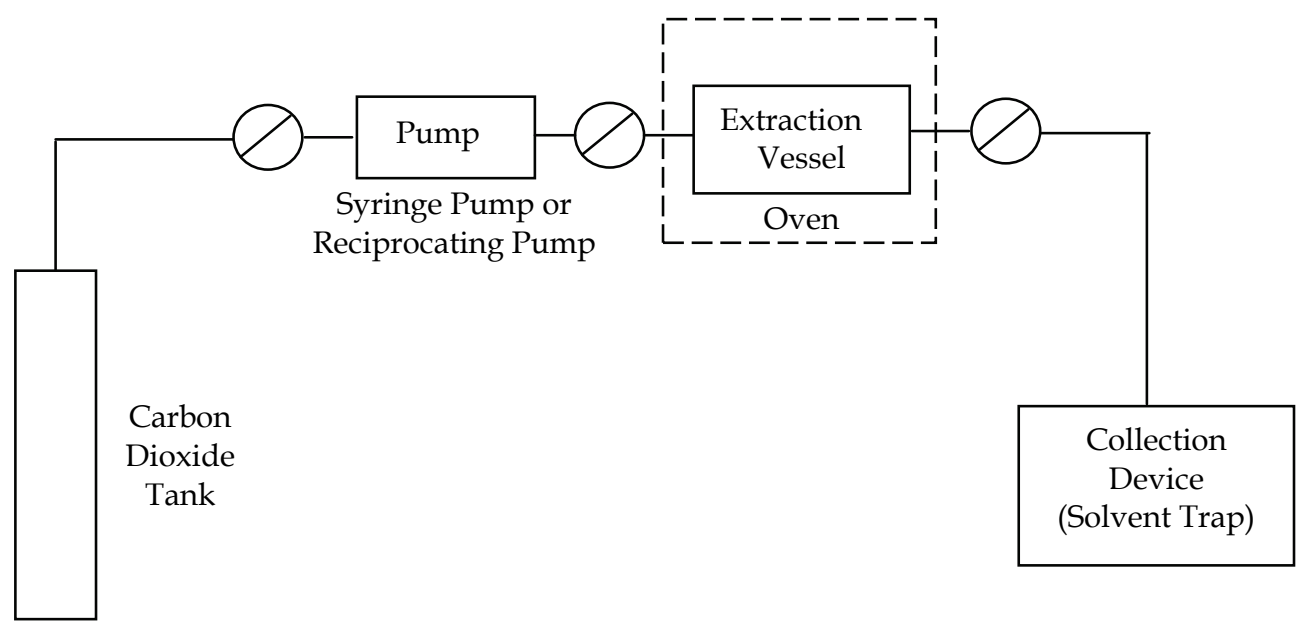

Fig. 7. Schematic Diagram of a SFE System (Fidalgo-Used et al., 2007)

Water is a poor choice for this technique because of its high critical temperature and pressure. The most widely used supercritical fluid is carbon dioxide which is characterized by low critical values and low chemical reactivity. Carbon dioxide is easily obtained in extremely pure form at a reasonable cost, and it has low viscosity, non-inflammable, environment-friendly, high diffusion rate with a high volatility and it can be separated from the collected analytes without incurring disposal problems. Nitrous oxide would be a good supercritical fluid, but it is highly inflammable. Ammonia is a polar substance with good solvent strength, but it is chemically reactive and corrosive. The hydrocarbons are usually inflammable and are not viable for analytical SFE. A common practice in SFE, which has to 
be taken into consideration in connection with the physicochemical properties of supercritical fluids, is the use of modifiers (co-solvents). There are compounds that are added to the primary fluid to enhance extraction efficiency. For example, the addition of a small percentage $(1-10 \%)$ of methanol to carbon dioxide expands its extraction range to include more polar analytes (Buldine et al., 2002).

King et al. (1993) applied SFE with carbon dioxide for the selective isolation of organochlorine, organophosphorus and organonitrogen pesticides from contaminated cereals. The resulting extracts were cleaned-up by GPC and GC-FPD and used for quantification. A determination method for 56 different pesticides was reported by Lehotay and Garcia (1997). The sample was frozen and a drying agent consisting of magnesium sulfate was mixed and homogenized with a small amount of dry ice. The sample was extracted with supercritical $\mathrm{CO}_{2}$, trapped with $\mathrm{C}_{18}$ bonded silica, eluted with acetone, and subsequently analyzed by GC ion-trap mass spectrometry. Magnesium sulfate as a drying agent was mixed with the sample to get rid of water, and gave a high recovery for methamidophos as well as for other pesticides.

Highly polar pesticides such as the phosphorothioates and phosphoramidothioates showed very low recoveries by the supercritical $\mathrm{CO}_{2}$ extraction method (e.g., acephate, omethoate and vamidothion). Generally, a modifier is added to the supercritical $\mathrm{CO}_{2}$ to improve the extraction yield. Stefani et al. (1997) worked on many extraction methods using two steps, such as two subsequent extractions of the same sample without the addition of a polar solvent to supercritical $\mathrm{CO}_{2}$. The two steps were similar except for the volume of the trap solvent. Celite and anhydrous calcined sodium sulfate were added as drying agents to the samples. The optimization of SFE on several organochlorine and organophosphorus pesticides in samples with high water content such as strawberry was performed. Lyophilization and addition of anhydrous sodium sulfate were examined to solve the problem caused by the water content of vegetable samples (Nerin et al., 1998). SFE efficiency is affected by a wide range of parameters such as the nature of the supercritical fluid, temperature and pressure, extraction time, the shape of the extraction cell, the sample particle size, the matrix type, the moisture content of the matrix and the analyte collection system. Due to these numerous parameters affecting the extraction efficiencies, SFE affords a high degree of selectivity and the extracts are relatively clean. However, the presence of water and fat in food samples can require extensive sample preparation and the development of more on-line cleanup procedures for SFE should enable further applications for food analysis to be developed. For example, sorbents, such as alumina, florisil and silica, can be placed in the extraction cell, or used as a cleanup following extraction to increase selectivity. Sorbents in the extraction cell can also be used for 'inverse' SFE extraction, in which interfering compounds are removed by a weak supercritical extraction fluid, leaving the analyte trapped on the sorbent for subsequent extraction under stronger conditions (King, 1998). Besides, the need to control so many operating parameters makes SFE optimization tedious and difficult in practice. Other disadvantages of the SFE technique include: limited sample size and high cost of the equipment.

\section{References}

Abhilash, P.C., Jamil, S. and Singh N. (2007). Matrix solid-phase dispersion extraction versus solid-phase extraction in the analysis of combined residues of hexachlorocyclohexane isomers in plant matrices. J. Chromatogr. A. 1176. 43-47. 
Ahmadi, F., Assadi, Y., Hosseini, S.M.R.M. and Rezaee, M. (2006). Detemination of organophosphorus pesticides in water samples by single drop microextraction and gas chromatography flame photometric detector. J. Chromatogr. A. 1101.307-312.

Amvrazia, E.G. \& Tsiropoulos, N.G. (2009a). Application of single-drop microextraction coupled with gas chromatography for the determination of multiclass pesticides in vegetables with nitrogen phosphorus and electron capture detection. J. Chromatogr. A. 1216. 2789-2797.

Anthemidis, A.N. and Ioannou, K.-I.G. (2009). On-line sequential injection dispersive liquidliquid microextraction system for flame atomic absorption spectrometric determination of copper and lead in water samples. Talanta 79. 86-91.

Arthur, C.L. and Pawliszyn, J. (1990), Solid Phase Microextraction with Thermal Desorption Using Fused Silica Optical Fibers, Anal. Chem., 62, 2145-2148.

Baltussen, E., Sandra, P., David, F. and Cramers, C. (1999). Stir bar sorptive extraction (SBSE), a novel extraction technique for aqueous samples: theory and principles. J. Mircrocolumn Separations. 11. 737-747.

Barker, S.A. (2000a). Application of matrix solid-phase dispersion in food analysis. J. Chromatogr. A. 880. 63-68.

Barker, S.A. (2000b). Matrix solid-phase dipersion - a review. J. Chromatogr. A. 885. 115-127.

Barker, S.A. (2007). Matrix solid phase dispersion (MSPD) - a review. J. Biochem. Biophys. Methods. 70. 151-162.

Barriada-Pereira, M., Concha-Grana, E., Gonzalez-Castro, M.J., Munitategui-Lorenzo, S., Lopez-Mahia, P., Prada-Rodriquez, D. and Fernandez-Fernandez. E. (2003). Microwave-assisted extraction versus soxhlet extraction in the analysis of 21 organochlorine pesticides in plants. J. Chromatogr. A. 1008. 115-122.

Barriada-Pereira, M., Gonzalez-Castro, M.J., Muniategui-Lorenzo, S., Lopex-Mahia, P., Prada-Rodriquez, D. and Fernandez-Fernandez, E. (2004) Determination of 21 organochorine pesticides in tree leaves using solid-phase extraction clean-up cartridges. J. Chromatogr. A. 1061. 133-139.

Beltran, J., Peruga, A., Pitarch, E., Lopez, F.J. and Hernandez, F. (2003). Application of solidphase microextraction for the determination of pyrethroid residues in vegetable samples by GC-MS. Anal. Bioanal. Chem. 376. 502-511.

Berijani, S., Assadi, Y., Anbia, M., Milani Hosseini, M.R. and Aghaee, E. (2006). Dispersive liquid-liquid microextraction combined with gas chromatography-flame photometric detection: Very simple, rapid and sensitive method for the determination of organophosphorus pesticides in water. J. Chromatogr. A, 1123. 19.

Bicchi, C., Cordero, C., Liberto, E., Rubiolo, P., Sgorbini, B. David, F. and Sandra, P. (2005). Dual-phase twisters: A new approach to headspace sorptive extraction and stir bar sorptive extraction. J. Chromatogr. A, 1094, 9-16.

Blasco, C., Font, G. and Pico, Y. (2004). Determination of dithiocarbamates and metabolites in plants by liquid chromatography-mass spectrometry. J. Chromatogr. A. 1028. 267276.

Bogialli, S. and Corcia, A.D. (2007). Matrix solid-phase dispersion as a valuable tool for extracting contaminants from foodstuffs - a review. J. Biochem. Biophys. Methods. 70. 163-179.

Bogialli, S., Curini, R., Corcia A.D., Nazzari, M. and Tamburro, D. (2004). A simple and rapid assay for analyzing residues of carbamate insecticides in vegetables and fruits: hot water extraction followed by liquid chromatography-mass spectrometry. J. Agric. Food Chem. 52. 665-671. 
Buldini, P.L., Ricci, L. and Sharma, J.L. (2002). Recent applications of sample preparation techniques in food analysis - a review. J. Chromatogr. A. 975. 47-70.

Cai, C.P., Liang, M. and Wen, R.R. (1995). Rapid multiresidue screening method for organophosphate pesticides in vegetables. Chromatograhia. 40. 417-420.

Cai, L., Xing, J., Dong, L. and Wu, C. (2003). Application of polyphenylmethylsiloxane coated fiber for solid-phase microextraction combined with microwave-assisted extraction for the determination of organochlorine pesticides in Chinese teas. $J$. Chromatogr. A. 1015. 11-21.

Cai, LS., Gong, SL., Chen, M. and Wu, CY. (2006). Vinyl crown ether as a novel radical crosslinked sol-gel SPME fiber for determination of organophosphorus pesticides in food samples. Anal. Chim. Acta, 559, 89-96.

Castro, J. Perez, R.A., Miguel, E., Sanchez-Brunete, C. and Tadeo, J.L. (2002). Analysis of endosulfan isomers and endosulfan sulfate in air and tomato leaves by gas chromatography with electron-capture detection and confirmation by gas chromatography-mass spectrometry. J. Chromatogr. A, 947, 119-127.

Chafer-Pericas, C., Herraez-Hernandez, R. and Campins-Falco, P. (2006). On-fibre solidphase microextraction coupled to conventional liquid chromatography versus intube solid-phase microextraction coupled to capillary liquid chromatograhy for the screening analysis of triaznies in water samples. J. Chromatogr. A. 1125. 159-171.

Chai, M.K. and Tan, G.H. (2009). Validation of a headspace solid-phase microextraction procedure with gas chromatography-electron capture detection of pesticide residues in fruits and vegetables. Food Chem. 117. 561-567.

Chai, M.K., Tan, G.H. and Asha, L. (2008) Optimization of headspace solid-phase microextraction for the determination of pesticide residues in vegetables and fruits. Analytical Sciences 24 (2). 273-276.

Chienthavorn, O., Pengpumkiat, S., Noomhorm, A. and Smith, R.M. (2007). Superheated water extraction and phase transfer methylation of phenoxy acid herbicides from solid matrices. J. Chromatogr. A, 1152. 268-273.

Chuang, J.C., Hart, K., Chang, J.S., Boman, L.E., Van Emon, J.M., and Reed, A.W. (2001). Evaluation of analytical methods for determining pesticides in baby foods and adult duplicate-diet samples. Anal. Chim. Acta 444, 87-95.

Cortés-Aguadoa, S., Sánchez-Moritoa, N., Arrebolab, F.J., Garrido Frenichb, A. and Martínez Vidalb, J.L. (2008). Fast screening of pesticide residues in fruit juice by solid-phase microextraction and gas chromatography-mass spectrometry. Food Chem. 107. 13141325.

Cunha, S.C., Fernandes, J.O. and Oliveira, M.B.P.P. (2007). Determination of phosmet and its metabolites in olives by matrix solid-phase dispersion and gas chromatographymass spectrometry. Talanta 73. 514-522

Cunha, S.C., Fernandes, J.O. and Oliveira, M.B.P.P. (2009). Fast analysis of multiple pesticide residues in apple juice using dispersive liquid-liquid microextraction and multidimensional gas chromatography- mass spectrometry. J. Chromatogr. A, 1216. 8835-8844.

David, F. and Sandra, P. (2007). Stir bar sorptive extraction for trace analysis - a review. J. Chromatogr. A. 1152. 54-69.

Dean, J.R. (2000). Extraction of organic pollutants from environmental matrices : selection of extraction technique. Trends Anal. Chem. 19(9). 553-564.

Diagne, R.G., Foster, R.D. and Khan, S.U. (2002). Comparison of xoxhlet and microwave assisted extractions for the determination of fenitrothion residues in beans. J. Agric. Food Chem. 50. 3204-3207. 
Eisert, R. and Levsen, K. (1996). Solid-phase microextraction coupled to gas chromatography:a new method for the analysis of organic in water. J. Chormatogr. A, 733. 143-157.

Farahani, H., Norouzi, P., Dinarvand, R. and Ganjali, M.R. (2007). Development of dispersive liquid-liquid microextraction combined with gas chromatography-mass spectrometry as a simple, rapid and highly sensitive method for the determination of phthalate esters in water samples. J. Chromatogr. A, 1172.105-112.

Fattahi, N., Assadi, Y., Milani Hosseini, M.R. and Jahromi., E.Z. (2007). Determination of chlorophenols in water samples using simultaneous dispersive liquid-liquid microextraction and derivatization followed by gas chromatography-electroncapture detection. J. Chromatogr. A, 1157.23-29.

Fidalgo-Used, N., Blanco-Gonzalez, E. and Sanz-Medel A. (2007). Sample handling strategies for the determination of persistent trace orgnic contaminants from biota samples. Anal. Chim. Acta. 590. 1-16.

Fontanals, N., Marce, R.M. and Borrull, F. (2005). New hydroplilic materials for solid-phase extraction. Trends Anal. Chem. 24. 394-406.

Fu, L., Liu, X., Hu, J., Zhao, X., Wang, H. and Wang, X. (2009). Application of dispersive liquid-liquid microextraction for the analysis of triazophos and carbaryl pesticides in water and fruit juice samples. Anal. Chim. Acta. 632. 289-295.

Fuentes, E., Baez, M.E., and Quinones A. (2008). Suitability of microwave-assisted extraction coupled with solid-phase extraction for organophosphorus pesticide determination in olive oil. J. Chromatogr. A. 1207. 38-46.

Guan, W., Wang, Y., Xu, F., and Guan, Y. (2008). Poly(phthalazine ether sulfone ketone) as novel stationary phase for stir bar sorptive extraction of organochlorine compounds and organophosphorus pesticides. J. Chromatogr. A, 1177, 28-35.

Hennion, M.C. (1999). Solid-phase extraction: method development, sorbents and coupling with liquid chromatography - a review. J. Chromatogr. A. 856. 3-54.

Hernández, F., Pozo, O.J., Sancho, J.V., Bijlsma, L., Barreda, M. and Pitarch E. (2006). Multiresidue liquid chromatography tandem mass spectrometry determination of 52 non gas chromatography-amenable pesticides and metabolites in different food commodities. J. Chromatogr. A, 1109, 242-252.

Hernandez-Borges, J., Ravelo-Perez, L.M., Hernandez-Suarez, E.M., Carnero, A. and Rodriguez-Delgado M.A. (2008). Determination of Abamectin Residues in Avocado by microwave-assisted extraction and HPLC with fluorescrence detection. Chromatographia 67. 69-75.

Herrera, M.C., Prados-Rosales, R.C., Luque-Garcia, J.L., and Luque de Castro, M.D. (2002). Static-dynamic pressurized hot water extraction coupled to on-line filtration solid phase extraction-high performance liquid chromatography-post-column derivatization-fluorescence detection for the analysis of N-methylcarbamates in foods. Anal. Chim. Acta 463. 189-197.

$\mathrm{Hu}$, Y.Y., Zheng, P., He, Y.Z. and Sheng, G.P. (2005). Response surface optimization for determination of pesticide multiresidues by matrix solid-phase dispersion and gas chromatography. J. Chromatogr. A. 1098. 188-193.

Jansson, C., Pihlstrom, T., Osterdahl, B. G. and Markides, K. E. (2004). A new multi-residue method for analysis of pesticide residues in fruits and vegetables using liquid chromatography with tandem mass spectrometric detection. J. Chromatogr. A, 1023, 93-104.

Juan-Garcia, A., Manes, J., Font, G. and Pico, Y. (2004). Evaluation of solid-phase extraction and stir-bar sorptive extraction for the determination of fungicide residues at low - 
$\mathrm{mg} / \mathrm{kg}$ levels in grapes by liquid chromatography-mass spectrometry. J. Chromatogr. A. 1050. 119-127.

Juan-Garcia, A., Pico, Y. and Font, G. (2005). Capillary electrophoresis for analyzing pesticides in fruits and vegetables using solid-phase extraction and stir-bar sorptive extraction. J. Chromatogr. A. 1073. 229-236.

King, J.W., Hopper, M.L., Luchtefeld, R.G., Taylor, S.L. and Orton, W.L. (1993). Optimization of Experimental Conditions for the Supercritical Carbon Dioxide Extraction of Pesticide Residues from Grains. J. AOAC. 76 (4). 857-864.

King. J.W. (1998). Integration of sample cleanup methods into analytical supercritical fluid extraction. Am. Lab. 30. 46-58.

Kodba, Z. C., and Voncina, D.B. (2007). A Rapid Method for the Determination of Organochlorine, Pyrethroid Pesticides and Polychlorobiphenyls in Fatty Foods Using GC with Electron Capture Detection. Chromatographia. 66, 619-624.

Kozani, R.R., Assadi, Y., Shemirani, F., Hosseini, M.R.M, Jamali, M.R. Talanta, 2007, 72: 387393.

Kristenson, E.M., Haverkate, E.G.J., Slooten, C.J. and Ramos, L. (2001). Miniaturized automated matrix solid-phase dispersion extraction of pesticides in fruits followed by gas chromatographic-mass spectrometerix analysis. J. Chromatogr. A. 917. 277-286.

Kristenson, E.M., Ramos, L. and Brinkman, U.A.T. (2006). Recent advances in matrix solidphase dispersion. Trends Anal. Chem. 25(2). 96-111.

Labbozzetta, S., Valco, L., Bertocchi, P. and Manna, L. (2005). Focused microwave-assisted extraction and LC determination of the active ingredient in naproxen-based suppositories. J. Pharmaceutical Biomedical Analysis. 39. 463-468.

Lambropoulou, D.A. and Albanis T.A. (2003). Headspace solid-phase microextraction in combination with gas chromatography-mass spectrometry for the rapid screening of organophosphorus insecticide residues in strawberries and cherries. J. Chromatogr. A. 993. 197-203.

Lambropoulou, D.A., Psillakis, E., Albanis, T.A. and Kalogerakis, N. (2004). Single drop microextraction for the analysis of organophosphorus insecticides in water. Anal. Chim. Acta. 516. 205-211.

Lehotay, S.J. and Garcia, A.V. (1997). Evaluation of different solid-phase traps for automated collection and clean-up in the analysis of multiple pesticides in fruits and vegetables after superciritical fluid extraction. J.Chromatogr. A, 765. 69-84.

Lopez-Blanco, M.C., Blanco-Cid, S., Cancho-Grande, B. and Simal-Gandara, J. (2003). Application of single drop microextraction and comparison with solid phase microextraction and solid phase extraction for the determination of alpha and beta endosulfan in water samples by gas chromatography electron capture detection. $J$. Chromatogr. A. 984. 245-252.

Menezes-Filho, A. Santos, F.N. and Paula-Pereira, P.A. (2010). Development, validation and application of a methodology based on solid-phase micro extraction followed by gas chromatography coupled to mass spectrometry (SPME/GC-MS) for the determination of pesticide residues in mangoes. Talanta, 81, 346-354.

Mester, Z. and Sturgeon, R. (2005). Trace element speciation using solid phase microextraction - a review. Spectrochimica Acta. Part B. 60. 1243-1269.

Mitani, K., Narimatsu, S. and Kataoka, H. (2003). Determination of daidzein and genistein in soybean foods by atuomated on-line in-tube solid-phase microextraction coupled to high-performance liquid chromatography. J. Chromatogr. A. 986. 169-177.

Moreno, J.L.F., Liebanas, F.J.A., Frenich, A.G. and Vidal, J.L.M. (2006). Evaluation of different sample treatments for determining pesticide residues in fat vegetable 
matrices like avocado by low-pressure gas chromatography-tandem mass spectrometry. J. Chromatogr. A. 1111. 97-105.

Navalon, A., Prieto, A., Araujo, L. and Vilchez, J.L. (2002). Determination of pyrimethanil and kresoxim-methyl in green groceries by headspace solid phase microextraction and gas chromatography-mass spectrometry. J. Chromatogr. A. 975. 355-360.

Nerin, C., Batlle, R. and Cacho, J. (1998). Determination of pesticides in high -water-content samples by off-line supercritical fluid extraction-gas chromatography-electroncapture detection. J. Chromatogr. A. 795. 117-124.

Pawliszyn, J. (1997). Solid Phase Microextraction - Theory and Practise. Wiley - VCH.

Pawliszyn, J. (1999). Applications of Solid Phase Mircoextraction. The Royal Society of Chemistry. Hertfordshire, UK.

Poole, C.F. and Poole, S.K. (2010). Extraction of organic compounds with room temperature ionic liquids. J. Chromatogr. A, 1217. 2268-2286.

Prosen, H. and Zupancic-Kralj, L. (1999). Solid-phase microextraction. Trends Anal. Chem. 18(4). 272-282.

Radišić, M., Grujić, S., Vasiljević T. and Lauševic, M. (2009). Determination of selected pesticides in fruit juices by matrix solid-phase dispersion and liquid chromatography-tandem mass spectrometry. Food Chem. 113, 712-719.

Ramos, J.J., Rial-Otero, R., Ramos, L. and Capelo, J.L. (2008). Ultrasonic-assisted matrix solid-phase dispersion as an improved methodology for the determination of pesticides in fruits. J. Chromatogr. A. 1212. 145-149.

Ramos, L., Kristenson, E.M. and Brinkman, U.A.H. (2002). Current use of pressurised liquid extraction and subcritical water extraction in environmental analysis - a review. J. Chromatogr. A. 975. 3-29.

Ravelo-Pe'rez, L.M., Herna'ndez-Borges, J., Asensio-Ramos, M. and Rodríguez-Delgado, M.A. (2009a). Ionic liquid based dispersive liquid-liquid microextraction for the extraction of pesticides from bananas. J. Chromatogr. A, 1216. 7336-7345.

Ravelo-Pe'rez, L.M., Herna'ndez-Borges, J., Herrera-Herrera, A.V. and Rodríguez-Delgado, M.A. (2009b). Pesticide extraction from table grapes and plums using ionic liquid based dispersive liquid-liquid microextractio. Anal. Bioanal. Chem. 395. 2387-2395.

Rezaee, M., Assadi, Y., Millani Hosseini, M.R., Aghaee, E., Ahmadi, F. and Berijani, S. (2006). Determination of organic compounds in water using dispersive liquid-liquid microextraction. J. Chromatogr. A, 1116. 1-9.

Roos, A.H., Munsteren, A.J.V., Nab, F.M. and Tuinstra, L.g.M.t. (1987). Universal extraction / cleanup procedure for screening of pesticides by extraction with ethyl acetate and size exclusion chromatography. Anal. Chim. Acta. 196. 95-102.

Sagratini, G., Mañes, J., Giardiná, D., Damiani, P., and Picó, Y. (2007). Analysis of carbamate and phenylurea pesticide residues in fruit juices by solid-phase microextraction and liquid chromatography-mass spectrometry. J. Chromatogr. A, 1147, 135-143.

Sandra, P., Tienpont, B. and David, F. (2003). Multi-residue screening of pesticides in vegebles, fruits and baby food by stir bar sorptive extraction-thermal desorptioncapillary gas chromatography-mass spectrometry. J. Chromatogr. A. 1000. 299-309.

Sharif, Z., Man, Y.B.C., Hamid N.S.A. and Chin, C.K. (2006). Determination of organochlorine and pyrethroid pesticides in fruit and vegetables using solid phase extraction clean-up catridges. J. Chromatogr. A. 1127. 254-261.

Shimelis, O., Yang, Y., Stenerson, K., Kaneko, T. \& Ye, M. (2007). Evaluation of a solid-phase extraction dual-layer carbon/primary secondary amine for clean-up of fatty acid matrix components from food extracts in multiresidue pesticide analysis. $J$. Chromatogr. A, 1165, 18-25. 
Silva, M.G.D., Aquino, A., Dorea, H.S. and Navickiene, S. (2008). Simultaneous determination of eight pesticide residues in coconut using MSPD and GC/MS. Talanta 76. 680-684.

Simplicio, A.L. and Boas, L.V. (1999). Validation of a solid-phase microextraction method for the determination of organophosphorus pesticides in fruits and fruit juice. $J$. Chromatogr. A. 833. 35-42.

Smith, R.M. (2002). Extractions with superheated water - A review. J. Chromatogr. A. 975. 31-46.

Smith, R.M. (2003). Before the injection-modern methods of sample preparation for separation techniques. J. Chromatogr. A. 1000. 3-27.

Štajnbaher, D. and Zupančič-Kral, L. (2003). Multiresidue method for determination of 90 pesticides in fresh fruits and vegetables using solid-phase extraction and gas chromatography-mass spectrometry. J. Chromatogr. A. 1015. 185-198.

Štajnbaher, D. and Zupančič-Kral, L. (2008). Optimisation of programmable temperature vaporizer-based large volume injection for determination of pesticide residues in fruits and vegetables using gas chromatography-mass spectrometry. J. Chromatogr. A. 1109. 316-326.

Stefani, R., Buzzi, M. and Grazzi, R. (1997). Supercritical fluid extraction of pesticide residues in fortified apple matrices. J. Chromatogr. A. 782. 123-132

Tao, S., Guo, L.Q., Wang, X.J., Liu, W.X., Ju, T.Z., Dawson, R., Cao, J., Xu, F.L. and Li, B.G. (2004). Use of sequential ASE extraction to evaluate the bioavailability of DDT and its metabolites to wheat roots in soils with various organic carbon contents. The Sc. the Total Environ. 320.1-9.

Thurman, E.M. and Mills, M.S. (1998). Solid-phase Extraction - Principle and Practice. John Wiley \& sons, INC.

Torres, C.M., Pico, Y. and Manes, J. (1996). Determination of pesticide residues in fruit and vegetables - a review. J. Chromatogr. A. 754. 301-331.

Xiao, Q., Hu, B., Yu, C., Xia, L. and Jiang, Z. (2006). Optimization of a single drop microextraction procedure for the determination of organophosphorus pesticides in water and fruit juice with gas chromatography-flame photometric detection. Talanta. 69. 848-855.

Xu, L., Basheer, C. and Lee, H.K. (2007). Developments in single-drop microextraction. J. Chromatogr. A. 1152. 184-192.

Zambonin, C.G., Quinto, M., Vietra, N.D. and Palmisano, F. (2004). Solid-phase microextraction-gas chromatography mass spectrometry: A fast and simple screening method for the assessment of organophosphorus pesticides residues in wine and fruit juices. Food Chem. 86. 269-274.

Zhang, M., Huang, J., Wei, C., Yua, B., Yang, X. \& Chen, X. (2008). Mixed liquids for singledrop microextraction of organochlorine pesticides in vegetables. Talanta 74. 599-604.

Zhao, E., Zhao, W., Han, L., Jiang, S. and Zhou, Z. (2007). Application of dispersive liquidliquid microextraction for the analysis of organophosphorus pesticides in watermelon and cucumber. J. Chromatogr. A, 1175. 137-140.

Zhao, L.M. and Lee, H.K. (2001). Application of static liquid-phase microextraction to the analysis of organochlorine pesticides in water. J. Chromatogr. A. 919. 381-388. 


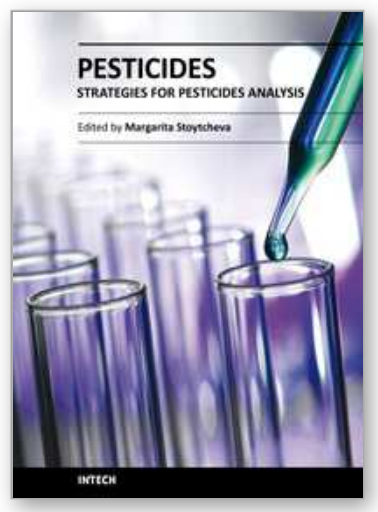

\author{
Pesticides - Strategies for Pesticides Analysis \\ Edited by Prof. Margarita Stoytcheva
}

ISBN 978-953-307-460-3

Hard cover, 404 pages

Publisher InTech

Published online 21, January, 2011

Published in print edition January, 2011

This book provides recent information on various analytical procedures and techniques, representing strategies for reliability, specificity, selectivity and sensitivity improvements in pesticides analysis. The volume covers three main topics: current trends in sample preparation, selective and sensitive chromatographic detection and determination of pesticide residues in food and environmental samples, and the application of biological (immunoassays-and biosensors-based) methods in pesticides analysis as an alternative to the chromatographic methods for "in situ" and "on line" pesticides quantification. Intended as electronic edition, providing immediate "open access" to its content, the book is easy to follow and will be of interest to professionals involved in pesticides analysis.

\title{
How to reference
}

In order to correctly reference this scholarly work, feel free to copy and paste the following:

Guan Huat Tan and Mee-Kin Chai (2011). Sample Preparation in the Analysis of Pesticides Residue in Food by Chromatographic Techniques, Pesticides - Strategies for Pesticides Analysis, Prof. Margarita Stoytcheva (Ed.), ISBN: 978-953-307-460-3, InTech, Available from: http://www.intechopen.com/books/pesticidesstrategies-for-pesticides-analysis/sample-preparation-in-the-analysis-of-pesticides-residue-in-food-bychromatographic-techniques

\section{INTECH}

open science | open minds

\author{
InTech Europe \\ University Campus STeP Ri \\ Slavka Krautzeka 83/A \\ 51000 Rijeka, Croatia \\ Phone: +385 (51) 770447 \\ Fax: +385 (51) 686166 \\ www.intechopen.com
}

\author{
InTech China \\ Unit 405, Office Block, Hotel Equatorial Shanghai \\ No.65, Yan An Road (West), Shanghai, 200040, China \\ 中国上海市延安西路65号上海国际贵都大饭店办公楼 405 单元 \\ Phone: +86-21-62489820 \\ Fax: +86-21-62489821
}


(C) 2011 The Author(s). Licensee IntechOpen. This chapter is distributed under the terms of the Creative Commons Attribution-NonCommercialShareAlike-3.0 License, which permits use, distribution and reproduction for non-commercial purposes, provided the original is properly cited and derivative works building on this content are distributed under the same license. 